\title{
INTEGRALGLEICHUNGEN UND DARSTELLUNG WILLKÜRLICHER FUNKTIONEN VON ZWEI VARIABELN.
}

Von Adolf Kneser (Breslau).

Adunanza del it giugno 1908 .

Die Schwingungen einer ebenen elastischen Membran und die Wärmeverteilung in einer ebenen leitenden Platte führen auf die folgende Randwertaufgabe: Es sei $G$ ein ebenes Gebiet, $x$ und $y$ rechtwinklige Koordinaten, $\lambda$ eine noch $z u$ bestimmende Konstante; dann soll auf der Fläche $G$ eine solche Grösse $\varphi(x, y)$ bestimmt werden, dass eine Differentialgleichung

$$
\Delta \varphi+\lambda \varphi=0, \quad \frac{\partial^{2} \varphi}{\partial x^{2}}+\frac{\partial^{2} \varphi}{\partial y^{2}}+\lambda \varphi=0
$$

besteht und am Rande, dessen innere Normale $n$ sei, die Gleichung

$$
\frac{d \varphi}{d n}-b \varphi=0
$$

gilt. Dabei sei $b$ eine gegebene positive Konstante, die auch die Werte o und $\infty$ annehmen kann; der letztere Fall bedeutet natürlich, dass die Randbedingung in der Form

$$
\varphi=0
$$

angesetzt ist. Die so bestimmten Funktionen $\varphi$ sind die Normalfunktionen oder Eigenfunktionen der betreffenden Aufgabe, $\lambda$ die zugehörigen Eigenwerte und es ist, um die Lösung des physikalischen Problems als allgemein nachzuweisen, nötig, eine auf der Fläche $G$ beliebig gegebene Funktion $f(x, y)$ in eine Reihe von der Form

$$
f(x, y)=\sum A \varphi(x, y),
$$

in der die $A$ Konstante bedeuten, zu entwickeln.

Von dieser Entwicklung hat STEKLofF $^{1}$ ) bewiesen, dass sie immer möglich ist, wenn die Funktion $f(x, y)$ stetig ist, stetige erste und zweite Ableitungen besitzt und am Rande des Gebietes $G$ derselben Randbedingung unterworfen ist wie die Eigenfunktionen. Dies wichtige Resultat kann aber noch nicht als abschliessend gelten,

I) Sur les problemes fondamentaux de la Physique mathématique [Annales scientifiques de l'École Normale supérieure (Paris), Ser. III, Bd. XIX (1902), S. 19I-259], S. 257. 
sondern bedarf auch vom physikalischen Standpunkte aus der Ergänzung. Denn bei der Wärmeleitungsaufgabe ist die dargestellte Funktion die Anfangstemperatur und es liegt im allgemeinen kein Grund vor, anzunehmen, dass diese der Randbedingung, die von dem Strahlungsvorgange bestimmt wird, genügt; z. B. eine konstante Anfangstemperatur wird der Randbedingung im allgemeinen nicht unterworfen sein. Auch unstetige Funktionen wird man zulassen müssen, da gerade praktisch wichtige Aufgaben der Wärmeleitung erfordern, dass man die Platte zu Anfang der untersuchten Vorgänge aus zwei verschieden temperierten Stücken zusammensetzt. Die Lücke, die hiernach die Theorie der Entwicklung willkürlicher Funktionen von zwei Variabeln nach den Eigenfunktionen der bezeichneten Probleme noch darbietet, wollen wir in dem speziellen Fall, dass das Gebiet $G$ kreisförmig ist, auszufüllen suchen.

Setzen wir den Radius dieses Kreises $=1$, so folgt aus der Differentialgleichung

$$
\Delta \varphi+\lambda \varphi=0
$$

indem man Polarkoordinaten $r, \theta$ einführt, in bekannter Weise ${ }^{2}$ ), dass die Eigenfunktionen die Gestalt

$$
J_{m}(k r) \cos m \theta, \quad J_{m}(k r) \sin m \theta
$$

haben, wobei $m$ eine nicht negative ganze Zahl, $k$ eine Konstante ist, die erst durch die Randbedingung bestimmt wird, und in der Differentialgleichung $\lambda=k^{2}$ zu setzen ist. Die Randbedingung vereinfacht sich dadurch, dass die Identität

gilt. Aus der ursprünglichen Form

$$
\frac{d}{d n}=-\frac{d}{d r}
$$

$$
\begin{array}{cc}
\frac{d \varphi}{d n}-b \varphi=0 \\
\text { geht also die Gleichung } \quad k J_{m}^{\prime}(k)+b J_{m}(k)=0
\end{array}
$$

hervor. Im Falle $b=\infty$ erhält man natürlich

$$
J_{m}(k)=0 \text {. }
$$

Es seien $k_{m_{\mathrm{I}}}, k_{m_{2}}, \ldots$ die positiven Wurzeln dieser Gleichung in wachsender Reihenfolge; sie sind in die angegebenen Ausdrücke der Eigenfunktionen an Stelle von $k$ einzusetzen, sodass man zu jedem Eigenwert zwei wesentlich verschiedene zugehörige Eigenfunktionen erhält, denen natürlich beliebige lineare Kombinationen mit konstanten Koeffizienten beigesellt werden können.

Nach den Funktionen

$$
J_{m}\left(k_{m \mu} r\right) \cos m \theta, \quad J_{m}\left(k_{m p} r\right) \sin m \theta
$$

können nun, und das ist das neue Resultat der vorliegenden Abhandlung; auch Funktionen entwickelt werden, die auf der Kreisfäche $G$ gegebene Unstetigkeiten von ziemlich

2) Riemann-Weber, Die partiellen Differentialgleichungen der mathematischen Physik, Bd. II (1901), S 104, S. 26r. 
allgemeinem Charakter darbieten und der Randbedingung nicht unterworfen sind. Damit erhalten wir Analoga zu den tiefer liegenden Sätzen in der Theorie der Fou. RIER'schen Reihen, die sich auf die Darstellung unstetiger Funktionen beziehen, und mit Hilfe der Sätze von P. DU Bors-Reymond zu den allgemeinsten Klassen durch die FourIER'schen Reihen darstellbarer Funktionen führen. Die entsprechenden Entwicklungen für Funktionen mehrerer Variabeln, die einer späteren Gelegenheit vorbehalten bleiben mögen, würden die kürzlich von DiNI ${ }^{3}$ ) veröffentlichten Sätze zu benutzen haben, die den von DU Bors-REYmond gegebenen analog sind.

$$
\$ I \text {. }
$$

\section{Einige auf die Besselschen Funktionen beztigliche Formeln.}

Die BesseL'sche Funktion $J_{m}(k \sqrt{x})$, in der $k$ eine Konstante, $m$ eine ganze Zahl bedeutet, ist ein Integral der Gleichung

$$
\frac{d}{d x}\left(4 x \frac{d y}{d x}\right)+\left(k^{2}-\frac{m^{2}}{x}\right) y=0
$$

das an der Stelle $x=\mathrm{o}$ endlich ist, an der Stelle $x=\mathrm{I}$ aber, da die Gleichung

gilt, die Relation

$$
\frac{d y}{d x}=\frac{k}{2 \sqrt{x}} J_{m}^{\prime}(k \sqrt{x})
$$

$$
2 \frac{d y}{d x}+b y=0
$$

erfüllt. Setzt man die erste oder zweite der Gleichungen

$$
u=\frac{x}{\xi}, \quad u=\frac{\xi}{x}
$$

an, jenachdem $\xi>x$ oder $x>\xi$, setzt man ferner im Falle $m=0, b>0$

und im Falle $m>0, b \gg 0$

$$
K(x, \xi)=\frac{\mathrm{I}}{2 b}-\frac{\mathrm{I}}{8} \log \frac{x \xi}{u}
$$

$$
K(x, \xi)=\frac{\mathrm{I}}{4 m}\left[u^{\frac{m}{2}}-(x \xi)^{\frac{m}{2}}\right]+\frac{(x \xi)^{\frac{m}{2}}}{2(m+b)},
$$

so sind die Funktionen $K$ symmetrisch in ihren beiden Argumenten, und die Grössen $J_{m}\left(k_{m \mu} \sqrt{x}\right)$ sind Lösungen der Integralgleichung

$$
\varphi \xi=k_{m \mu}^{2} \int_{0}^{1} K(x, \xi) \varphi x . d x
$$

3) Sugli integrali multipli in generale, e su quelli che valgono per la rappresentazione analitica delle funzioni di piu variabili reali [Diese Rendiconti, Bd. XVIII (1904), S. 318-359]. 
wie man direkt verifizieren kann; $\varphi x$ sind also die Eigenfunktionen des Kernes $K(x, \xi)$ und $k_{m \mu}^{2}$ die zugehörigen Eigenwerte. Erstere sind, wie man sagt, normiert, wenn die Gleichung

$$
\int_{0}^{1}(\varphi x)^{2} d x=\mathrm{I}
$$

gilt. Man erreicht dies offenbar durch passende Wahl der Grösse $C$ indem man

setzt.

$$
\varphi x=C J_{m}\left(k_{m \mu} \sqrt{x}\right)
$$

Die eingeführten Kerne haben alle Eigenschaften, die in einer früheren Untersuchung gefordert wurden ${ }^{4}$ ). Nach den am angeführten Ort gegebenen Entwicklungen werden daher die Formeln

$$
K(x, \xi)=\sum_{\mu} \frac{\varphi x \cdot \varphi \xi}{k_{m \mu}^{2}}, \quad \frac{\partial K(x, \xi)}{\partial x}=\sum_{\mu} \frac{\varphi^{\prime} x \cdot \varphi \xi}{k_{m \mu}^{2}}
$$

erwiesen sein, sobald folgende Tatsachen feststehen: Die Reihe

$$
\sum_{\mu} \frac{\varphi x \cdot \varphi \xi}{k_{m \mu}^{4}}
$$

konvergiert gleichmässig, wenn $x$ und $\xi$ die Strecke von o bis I durchlaufen; dasselbe gilt von der Reihe

$$
\sum_{\mu} \frac{\varphi^{\prime} x \cdot \varphi \xi}{k_{m \mu}^{4}}
$$

wobei aber der Wert $x=0, \xi=0$ ausgeschlossen sein kann; endlich konvergiert die Reihe

$$
\sum_{\mu} \frac{\varphi x \cdot \varphi \xi}{k_{m \mu}^{2}}
$$

gleichmässig, wenn $x$ und $\xi$ beliebige Teilstrecken des Intervalls von o bis I durchlaufen, die aber den Punkt o nicht enthalten. Der Beweis lässt sich für einen beliebigen Wert von $m$ genau so führen, wie ich ihn in $\ 7$ der zitierten Abhandlung für $m=0$ durchgeführt habe; denn, worauf schon dort hingewiesen wurde, die wesentliche Grundlage, die asymptotische Darstellung der Besses'schen Funktionen durch trigonometrische, bleibt auch bei beliebigen Werten von $m$ gültig.

Um die hieraus folgenden Formeln aufzustellen, bilden wir explicite die normierten Eigenfunktionen unserer Kerne. Offenbar sind sie

$$
J_{m}\left(k_{m \mu} \sqrt{x}\right)\left[\int_{0}^{1} J_{m}\left(k_{m \mu} \sqrt{x}\right)^{2} d x\right]^{-\frac{1}{2}} .
$$

Bezeichnen wir daher die Grössen

$$
\int_{0}^{1} J_{m}\left(k_{m \mu} \sqrt{x}\right)^{2} d x=2 \int_{0}^{1} \alpha J_{m}\left(k_{m \mu} \alpha\right)^{2} d \alpha
$$

4) KNESER, Die Theorie der Integralgleichungen und die Darstellung willkürlicher Funktionen in der mathematischen Physik [Mathematische Annalen, Bd. LXIII (1907), S. 477-524], S. 486. 
durch $2 N_{m \mu}$, wenn $m$ positiv ist und durch $N_{m \mu}$ im Falle $m=0$, so sind die normierten Eigenfunktionen

$$
\frac{J_{\circ}\left(k_{\mathrm{o \mu}} \sqrt{x}\right)}{\sqrt{N_{\mathrm{o} \mu}}}, \frac{J_{m}\left(k_{m \mu} \sqrt{x}\right)}{\sqrt{2 N_{m \mu}}}, \quad m>0,
$$

und man kann folgende Formeln als bewiesen annehmen :

$$
\begin{gathered}
\frac{\mathrm{I}}{2 b}-\frac{\mathrm{I}}{8} \log \frac{x \xi}{u}=\sum_{\mu}^{\mathrm{I}, \infty} \frac{J_{\mathrm{o}}\left(k_{\mathrm{o} \mu} \sqrt{x}\right) J_{\mathrm{o}}\left(k_{\mathrm{o \mu}} \sqrt{\xi}\right)}{N_{\mathrm{o} \mu} k_{\mathrm{o} \mu}^{2}}, \\
\frac{\mathrm{I}}{4 m}\left[u^{\frac{m}{2}}-(x \xi)^{\frac{m}{2}}\right]+\frac{(x \xi)^{\frac{m}{2}}}{2(m+b)}=\sum_{\mu}^{\mathrm{I}, \infty} \frac{J_{m}\left(k_{m \mu} \sqrt{x}\right) J_{m}\left(k_{m \mu} \sqrt{\xi}\right)}{2 N_{m \mu} k_{m \mu}^{2}}, \quad m>0 ;
\end{gathered}
$$

indem man $x, \xi$ durch $x^{2}, \xi^{2}$ ersetzt, erhält man

$$
\begin{gathered}
\frac{\mathrm{I}}{2 b}-\frac{\mathrm{I}}{4} \log \frac{x \xi}{u}=K(x, \xi)_{0}, \\
\frac{\mathrm{I}}{2 m}\left[u^{m}-(x \xi)^{m}\right]+\frac{(x \xi)^{m}}{m+b}=K(x, \xi)_{m}, \quad m>0
\end{gathered}
$$

wobei allgemein die Bezeichnung

$$
K(x, \xi)_{m}=\sum_{\mu}^{\mathrm{r}, \infty} \frac{J_{m}\left(k_{m \mu} x\right) J_{m}\left(k_{m \mu} \xi\right)}{N_{m \mu} k_{m \mu}^{2}}, m \supseteq 0
$$

eingeführt ist; dann besteht nach den zitierten Sätzen auch die Gleichung

$$
\frac{\partial K(x, \xi)_{m}}{\partial x}=\sum_{\mu}^{\mathrm{r}, \infty} \frac{J_{m}^{\prime}\left(k_{m \mu} x\right) J_{m}\left(k_{m \mu} \xi\right)}{N_{m \mu} k_{m \mu}}
$$

Die erhaltenen Formeln gelten zunächst nur mit Ausschluss des Falles $n=b=0$. Für diesen können die entsprechenden Resultate, die wir nicht streng beweisen wollen, durch einen Grenzübergang wenigstens plausibel gemacht werden. Da nämlich annähernd

$$
J_{\mathrm{o}}(x)=\mathrm{I}-\frac{x^{2}}{4}
$$

gesetzt werden kann, ergibt sich aus der Bedingungsgleichung

der angenäherte Wert

$$
k J_{0}^{\prime}(k)+b J_{0}(k)=0
$$

$$
b=\frac{k^{2}}{2}, \quad \frac{\mathrm{I}}{2 b}=\frac{\mathrm{I}}{k^{2}} ;
$$

anderseits ist das bei unendlich abnehmenden Werten von $b$ im Ausdruck $K(x, \xi)_{0}$ unendlich werdende Glied, welches dem unendlich kleinen $k=k_{\text {o }}$ entspricht,

$$
\frac{\left(\mathrm{I}-\frac{k^{2} x^{2}}{4}\right)\left(\mathrm{I}-\frac{k^{2} \xi^{2}}{4}\right)}{2 k^{2} \int_{0}^{1}\left(\mathrm{I}-\frac{\alpha^{2} \xi^{2}}{4}\right)^{2} \alpha d x}
$$

Rend. Circ. Matem. Palermo, t. XXVII ( ${ }^{\circ}$ sem. 1909). - Stampato il 25 settembre Igo8. 
oder abgekürzt

$$
\frac{\mathrm{I}-\frac{k^{2}}{4}\left(x^{2}+\xi^{2}\right)}{k^{2}} .
$$

Indem man also das Glied $\frac{\mathrm{r}}{k^{2}}$ weglässt, ergibt sich bei der Annahme $b=0$

$$
\frac{x^{2}+\xi^{2}}{4}-\frac{1}{4} \log \frac{x \xi}{u}=\sum_{\mu} \frac{J_{o}\left(k_{\mathrm{o \mu}} x\right) J_{\mathrm{o}}\left(k_{\mathrm{o} \mu} \xi\right)}{k_{\mathrm{o} \mu}^{2} N_{\mathrm{o} \mu}},
$$

wobei nun rechts über alle von Null verschiedenen Werten $k_{\text {ou }}$ zu summieren ist.

Wir fügen hier noch eine Eigentümlichkeit der Reihen $K(x, \xi)_{m}$ hinzu, von der wir später Gebrauch machen werden. Obwohl diese Reihen offenbar nicht gleichmässig konvergieren, wenn $x$ das Intervall von o bis $\mathrm{I}$ durchläuft, so haben sie doch die Eigenschaft, mit einer in diesem Intervall stetigen Funktion des Ortes multipliziert immer gliedweise integrierbar zu sein.

In der Tat folgt aus der asymptotischen Darstellung der Funktion $J_{m}$ für die Grössen $N_{m \mu}$ die Formel

$$
\frac{\mathrm{I}}{k_{m \mu}^{2} N_{m \mu}}=\frac{\Psi}{k_{m \mu}}
$$

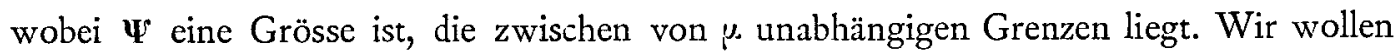
diesen Buchstaben auch fernerhin für andere Grössen von derselben Beschaffenheit, also vieldeutig gebrauchen.

Anderseits hat man, wenn $a$ und $b$ irgend welche Werte der Strecke von o bis I mit Einschluss der Grenzen sind,

$$
\int_{a}^{b} f \alpha \cdot J_{m}\left(k_{m \mu} \alpha\right) d \alpha=\int_{a}^{b} \frac{f \alpha}{\sqrt{\alpha}} \cdot \sqrt{\alpha} J_{m}\left(k_{m \mu} \alpha\right) d \alpha
$$

und da die Grössen $\sqrt{x} J_{m}(x)$ zwischen von $x$ unabhängigen Grenzen bleiben, sodass

gesetzt werden kann, das Integral

$$
\sqrt{x} J_{m}\left(k_{m \mu} x\right)=\frac{\Psi}{\sqrt{k_{m \mu}}}
$$

$$
\int_{a}^{b} \frac{d \alpha}{\sqrt{\alpha}}
$$

aber endlich bleibt, auch wenn die untere Grenze $a$ in den Wert o hineinrückt, so findet man

$$
\int_{a}^{b} f \alpha \cdot J_{m}\left(k_{m \mu} \alpha\right) d \alpha=\frac{\Psi}{\sqrt{k_{m \mu}}},
$$

wobei die Schranken der Grösse $\Psi$ auch von $a$ und $b$ unabhängig gewählt werden können.

Wenn ferner $\alpha$ eine Funktion eines Parameters $t$ ist, die einen stetigen, nur eine endliche Anzahl von Malen das Vorzeichen wechselnden Differentialquotienten besitzt, solange $t$ in einem gewissen Intervalle $T$ bleibt und wenn die Grösse $\alpha$ niemals die Gren- 
zen $\mathrm{o}$ und I, zwischen denen sie bleibt, überschreitet, so findet man zunächst für ein Integrationsintervall, in dem $\frac{d \alpha}{d t}$ ein festes Zeichen behält,

$$
\int_{a}^{b} f \alpha \cdot J_{m}\left(k_{m \mu} \alpha\right) \frac{d \alpha}{d t} d t=\frac{\Psi}{\sqrt{k_{m \mu}}} \int_{a}^{b} \frac{d t}{\sqrt{\alpha}} \frac{d \alpha}{d t}=\frac{\Psi}{\sqrt{k_{m \mu}}},
$$

und diese Formel bleibt auch gültig, wenn $\frac{d \alpha}{d t}$ eine endliche Anzahl von Malen das Zeichen wechselt, da man nur die Formeln von dieser Gestalt zu addieren braucht, die man für die einzelnen Intervalle aufstellen kann, in denen $\frac{d \alpha}{d t}$ das konstante Zeichen behält.

Hieraus folgt weiter unmittelbar, dass man setzen kann

$$
P_{\mu}^{a, b}=\frac{J_{m}\left(k_{m \mu} \xi\right)}{N_{m \mu} k_{m \mu}^{2}} \int_{a}^{b} f \alpha \cdot J_{m \mu}\left(k_{m \mu} \alpha\right) \frac{d \alpha}{d t} d t=\frac{\Psi}{k_{m \mu}^{\frac{5}{2}}} .
$$

Nun lehrt die bekannte asymptotische Darstellung der Funktion $J_{m}$ sofort, dass die Werte $k_{m \mu}$ bei wachsenden $\mu$ im wesentlichen wie die Glieder einer arithmetischen Progression fortschreiten. Die letzte Formel ergibt also, dass die Reihe

$$
\sum_{\mu}^{1, \infty} P_{\mu}^{a, b}
$$

bei beliebigen Werten von $a$ und $b$ im Intervall $T$ gleichmässig konvergiert, also eine stetige Funktion der Grenzen repräsentiert.

Anderseits gilt die Gleichung

$$
\sum_{\mu}^{1, \infty} P_{\mu}^{a, b}=\int_{a}^{b} f \alpha \cdot K(\alpha, \xi)_{m} \frac{d \alpha}{d t} d t
$$

jedenfalls, wenn das Integrationsintervall so beschränkt wird, dass die Variabele $\alpha$ um ein beliebig kleines festes Stück von o entfernt bleibt, da die Reihe $K(x, \xi)_{m}$, wie die asymptotische Darstellung der Bessel'schen Funktion lehrt, dann gleichmässig konvergiert. $\mathrm{Da}$ nun aber die rechte Seite der letzten Gleichung offenbar eine stetige Funktion von $a$ und $b$ ist, und dasselbe von der linken Seite soeben bewiesen wurde, so folgt, dass die Gleichung auch dann gilt, wenn innerhalb des Integrationsintervalls der Wert $\alpha=0$ erreicht wird.

Da endlich offenbar die Gleichung

$$
\int_{a}^{b} f \alpha_{.} J_{m}^{\prime}\left(k_{m \mu} \alpha\right) \frac{d \alpha}{d t} d t=\left.f \alpha_{.} J_{m}\left(k_{m \mu} \alpha\right)\right|_{t=a} ^{t=b}-\int_{a}^{b} f^{\prime} \alpha . J_{m}\left(k_{m \mu} \alpha\right) \frac{d \alpha}{d t} d t
$$

gilt, und die Funktion $f^{\prime} \alpha$ dieselben Eigenschaften hat, die wir bisher von $f \alpha$ gefordert und benutzt haben, so sieht man, dass in dem Integral

$$
\int_{\alpha}^{b} f \alpha \cdot \sum_{\mu}^{\mathrm{r}, \infty} \frac{J_{m}^{\prime}\left(k_{m \mu} \alpha\right) J_{m}\left(k_{m \mu} \xi\right)}{k_{m \mu}} N_{m \mu} \frac{d \alpha}{d t} d t
$$


gliedweise integriert werden kann: Anders ausgedrückt die Reihen

$$
\sum_{\mu}^{\mathrm{r}, \infty} \frac{J_{m}\left(k_{m \mu} \xi\right) J_{m}\left(k_{m \mu} \alpha\right)}{k_{m \mu}^{2} N_{m \mu}}, \quad \sum_{\mu}^{\mathrm{r}, \infty} \frac{J_{m}\left(k_{m \mu} \xi\right) J_{m}^{\prime}\left(k_{m \mu} \alpha\right)}{k_{m \mu} N_{m \mu}}
$$

können mit $f \alpha \cdot \frac{d \alpha}{d t} \cdot d t$ multipliziert unter den für $f \propto$ und $t$ geltenden Voraussetzungen gliedweise integriert werden.

\section{\2.}

\section{Die Integralgleichungen der betrachteten Probleme.}

Wir betrachten von jetzt ab ausschliesslich die Kreisfläche $G$ vom Radius I und bezeichnen ihr angehörige Stellen durch die Ziffern o, r, ..., die zugehörigen Polarkoordinaten durch $r_{0}, \theta_{0}, r_{1}, \theta_{1}, \ldots$ und die die betreffenden Punkte enthaltenden Flächen- und Linienelemente durch $d s_{0}, d s_{1}, \ldots, d l_{0}, d l_{1}, \ldots$ Dabei wollen wir uns vorbehalten, den Index o öfter einmal wegzulassen, sodass die Zeichen $r, \theta, d s, d l$ immer auf den Punkt o zu beziehen sind. Funktionen der Stellen o, I, ..., wie $F\left(r_{0}, \theta_{0}\right), F\left(r_{1}, \theta_{1}\right), \ldots$, bezeichnen wir kurz durch $F_{\mathrm{o}}, F_{\mathrm{I}}$, u. s. f.

Die Eigenfunktionen, die bei dem in der Einleitung bezeichneten Problem auftreten, nämlich

$$
J_{m}\left(k_{m \mu} r\right) \cos m \theta, \quad J_{m}\left(k_{m \mu} r\right) \sin m \theta
$$

wollen wir normieren, d.h. mit solchen konstanten Faktoren multiplizieren, dass das über die Fläche $G$ erstreckte Integral ihres Quadrats den Wert I erhält. Da offenbar nach $r$ von o bis $\mathrm{I}$, nach $\theta$ von o bis $2 \pi \mathrm{zu}$ integrieren ist, und die Gleichungen

$$
d s=r d r d \theta, \quad \int_{0}^{2 \pi} \sin ^{2} m \theta d \theta=\int_{0}^{2 \pi} \cos ^{2} m \theta d \theta=\pi, \quad m>0
$$

gelten, so erhält man, indem die Bezeichnungen

$$
\begin{aligned}
& N_{m \mathrm{k}}=\int_{0}^{1} \alpha J_{m}\left(k_{m \mu} \alpha\right)^{2} d \alpha, \quad m>0 \\
& N_{\mathrm{o \mu} \alpha}=2 \int_{0}^{1} \alpha J_{\circ}\left(k_{\mathrm{o} \mu} \alpha\right)^{2} d \alpha
\end{aligned}
$$

festgehalten werden, als normierte Eigenfunktionen, die $\mathrm{zu}$ dem Eigenwert $\lambda_{m \mu}=k_{m \mu}^{2}$ gehören, die Ausdrücke

$$
\varphi_{m \mu} \circ=\frac{J_{m}\left(k_{m \mu} r\right) \cos m \theta}{\sqrt{\pi N_{m \mu}}}, \quad \bar{\varphi}_{m \mu} \circ=\frac{J_{m}\left(k_{m \mu} r\right) \sin m \theta}{\sqrt{\pi N_{m \mu}}} .
$$

Die von FREDHOLM begründete Theorie der Integralgleichungen in der ihr von Schmidt ${ }^{5}$ ) gegebenen Gestalt regt nun dazu an, die über alle Kombinationen von $m$

5) Zur Theorie der linearen und nichtlinearen Integralgleichungen, I. TEIL : Entwicklung willkürlicher Funktionen nach Systemen vorgerschriebener [Mathematische Annalen, Bd. LXIII (1907), S. 433-476], S. 449. - Inauguraldissertation (Göttingen 190j). 
und $\mu$ zu erstreckende Summe

$$
\sum \frac{\varphi_{m \mu} 0 . \varphi_{m \mu} \mathrm{I}+\bar{\varphi}_{m \mu} 0 . \bar{\varphi}_{m \mu} \mathrm{I}}{\lambda_{m \mu}}
$$

zu bilden und man erwartet nit einer gewissen Wahrscheinlichkeit, sie dem Kerne $K(\mathrm{O}, \mathrm{I})$ der Integralgleichung, deren Eigenfunktionen die Grössen $\varphi, \bar{\varphi}$ sind, gleich zu finden.

Wir wollen die angedeutete Summation immer in einer besonderen, durch den Erfolg gerechtfertigten Reihenfolge vornehmen und setzen als Definition an

$$
K(\mathrm{o}, \mathrm{I})=\sum_{m}^{o, \infty} \sum_{\mu}^{\mathrm{I}, \infty} \frac{\varphi_{m \mu} \mathrm{O} \cdot \varphi_{m \mu} \mathrm{I}+\bar{\varphi}_{m \mu} \mathrm{o} \cdot \bar{\varphi}_{m \mu} \mathrm{I}}{\lambda_{m \mu}}
$$

wobei nach den angegebenen Ausdrücken der Grössen $\varphi$ und $\lambda$ gesetzt werden kann,

$$
\begin{aligned}
K(\mathrm{o}, \mathrm{I}) & =\sum_{m}^{0, \infty} \sum_{\mu}^{1, \infty} \frac{\cos m\left(\theta_{\mathrm{I}}-\theta\right) J_{m}\left(k_{m \mu} r\right) J_{m}\left(k_{m \mu} r_{1}\right)}{\pi N_{m \mu} k_{m \mu}^{2}} \\
& =\frac{\mathrm{I}}{\pi} \sum_{m}^{0, \infty} \cos m\left(\theta_{1}-\theta\right) \sum_{\mu}^{0, \infty} \frac{J_{m}\left(k_{m \mu} r\right) J_{m}\left(k_{m \mu} r_{1}\right)}{N_{m \mu} k_{m \mu}^{2}}
\end{aligned}
$$

oder, indem man das in $\subseteq \mathrm{I}$ eingeführte Zeichen $K(x, \xi)_{m}$ anwendet,

$$
=\frac{\mathrm{I}}{\pi} \sum_{m}^{\mathrm{o}, \infty} K\left(r, r_{\mathrm{1}}\right)_{m} \cos m\left(\theta_{\mathrm{r}}-\theta\right) \text {. }
$$

Wenn man die in $\ \mathrm{I}$ gegebenen Werte der Grössen $K_{m}$ benutzt und unter $u$ denjenigen der Werte $\frac{r}{r_{\mathrm{I}}}$ und $\frac{r_{\mathrm{I}}}{r}$ versteht, der nicht grösser als Eins ist, ergibt sich hieraus für den Fall $b>0$

$$
\pi K(\mathrm{o}, \mathrm{I})=\frac{\mathrm{I}}{2 b}-\frac{\mathrm{I}}{4} \log \frac{r r_{\mathrm{s}}}{u}+\sum_{m}^{\mathrm{I}, \infty} \frac{\cos m\left(\theta-\theta_{\mathrm{s}}\right)}{2 m}\left[u^{m}+\frac{m-b}{m+b}\left(r r_{\mathrm{x}}\right)^{m}\right] \text {, }
$$

für den Fall $b=$ o aber

$$
\pi K(\mathrm{o}, \mathrm{I})=\frac{r^{2}+r_{1}^{2}}{4}-\frac{\mathrm{I}}{4} \log \frac{r r_{\mathrm{I}}}{u}+\sum_{m}^{1, \infty} \frac{\cos m\left(\theta-\theta_{\mathrm{I}}\right)}{2 m}\left[u^{m}+\left(r r_{\mathrm{s}}\right)^{m}\right] .
$$

Die erste dieser Formeln behält offenbar auch im Falle $b=\infty$ einen bestimmten Sinn.

Hier ist nun der Umstand zu beachten, dass jede der Reihen

$$
\sum \alpha^{m} \cos m \beta, \quad \sum \alpha^{m} \sin m \beta, \quad \sum \frac{\alpha^{m} \cos m \beta}{m}, \quad \sum \frac{\alpha^{m} \sin m \beta}{m}
$$

der reelle oder imaginäre Teil einer leicht angebbaren Funktion des komplexen Arguments $\alpha e^{\beta i}$ sind und unter der Voraussetzung $\alpha<$ I gleichmässig konvergieren. Da ferner die Reihen

$$
\sum_{m}^{\mathrm{r}, \infty} \frac{\alpha^{m} \cos m \beta}{m+b}, \quad \sum \frac{\alpha^{-m} \cos m \beta}{m+b}
$$

der reelle und imaginäre Teil der Funktion

$$
z^{-b} \int_{0}^{\alpha} \frac{w^{h-1} d w}{1-w}
$$


sind, in der man von o nach $z$ längs eines geradlinigen Integrationsweges integriert und $z=\alpha^{ \pm \mathrm{r}} e^{\beta i}$ setzt, so sieht man, dass die Reihen unter der Bedingung $\alpha<\mathrm{I}$ konvergieren und gliedweise nach $\alpha$ und $\beta$ differenziert werden können. Endlich bedenke man, dass die elementaren Formeln

$$
\begin{gathered}
-\log \sqrt{\mathrm{I}-2 \alpha \cos \beta+\alpha^{2}}=\sum_{m}^{\mathrm{I}, \infty} \frac{\alpha^{m} \cos m \beta}{m}, \\
\sum_{m}^{\mathrm{s}, \infty} \frac{\alpha^{m} \cos m \beta}{m+b}=\left.\Re\left(z^{-h} \int_{0}^{\chi} \frac{w^{h-1} d w}{\mathrm{I}-w}\right)\right|^{\mathrm{z}=\alpha_{e} \beta i}
\end{gathered}
$$

auch im Falle $\alpha=$ I gelten, wenn nur $\cos \xi$ von I verschieden ist; mit ihrer Hilfe kann man die obigen Reihen für $K(\mathrm{o}, \mathrm{I})$ sämtlich summieren und findet bei positiven Werten $h$

$$
\begin{aligned}
K(\mathrm{o}, \mathrm{I})=\frac{\mathrm{I}}{2 \pi b} & +\frac{\mathrm{I}}{2 \pi} \log \sqrt{\frac{\mathrm{I}-2 r r_{\mathrm{I}} \cos \left(\theta-\theta_{\mathrm{I}}\right)+r^{2} r_{\mathrm{I}}^{2}}{r_{\mathrm{I}}^{2}-2 r r_{\mathrm{I}} \cos \left(\theta-\theta_{\mathrm{I}}\right)+r^{2}}} \\
+ & \frac{\mathrm{I}}{\pi} \Re\left[\left(r r_{\mathrm{I}} e^{i\left(\theta-\theta_{\mathrm{I}}\right)}\right)^{-b} \int_{0}^{r r_{\mathrm{I}} e^{\left(\theta-\theta_{1}\right) i}} \frac{w^{b-1} d w}{\mathrm{I}-w}\right],
\end{aligned}
$$

wobei das letzte Glied im Falle $b=\infty$ einfach wegzulassen ist; im Falle $b=0$ ergibt sich

$$
\begin{aligned}
K(\mathrm{o}, \mathrm{I})=\frac{r^{2}+r_{1}^{2}}{4 \pi} & -\frac{\mathrm{I}}{2 \pi} \log \sqrt{\mathrm{I}-2 r r_{\mathrm{I}} \cos \left(\theta-\theta_{\mathrm{I}}\right)+r^{2} r_{\mathrm{I}}^{2}} \\
& -\frac{\mathrm{I}}{2 \pi} \log \sqrt{r_{\mathrm{I}}^{2}-2 r r_{\mathrm{I}} \cos \left(\theta-\theta_{\mathrm{I}}\right)+r^{2}} .
\end{aligned}
$$

In beiden Fällen führt eine einfache Rechnung auf die Formel

$$
\frac{d K(\mathrm{o}, \mathrm{I})}{d n_{\mathrm{o}}}-b K(\mathrm{o}, \mathrm{I})=\mathrm{o}
$$

bei der Annahme $r_{\mathrm{o}}=\mathrm{x}$; d.h. die Grösse $K$ erfüllt die Randbedingung der Eigenfunktionen. Ebenso leicht erhält man, wenn allgemein

gesetzt wird, im Falle $b>0$

$$
\Delta_{\nu}=\frac{\partial^{2}}{\partial x_{v}^{2}}+\frac{\partial^{2}}{\partial y_{\nu}^{2}}
$$

und im Falle $b=0$

$$
\Delta_{\mathrm{o}} K(\mathrm{o}, \mathrm{I})=\Delta_{\mathrm{s}} K(\mathrm{o}, \mathrm{I})=\mathrm{o} \text {, }
$$

$$
\Delta_{\mathrm{o}} K(\mathrm{o}, \mathrm{I})=\Delta_{\mathrm{r}} K(\mathrm{o}, \mathrm{I})=\frac{\mathrm{I}}{\pi} \text {. }
$$

Aus den erhaltenen Ausdrücken ersieht man ferner, dass die Funktion $K(\mathrm{o}, \mathrm{I})$ nur dann aufhört, mit ihren Ableitungen endlich und stetig zu sein, wenn die Punkte o und I zusammenrücken; bei dieser Annahme geht sie im wesentlichen in den Ausdruck

$$
-\frac{1}{2 \pi} \log P_{\text {or }}
$$

über, indem durch $\rho_{o 1}$ der Abstand der Punkte o und I, d.h. die Grösse

$$
\rho_{01}=\sqrt{r_{1}^{2}-2 r r_{1} \cos \left(\theta-\theta_{1}\right)+r^{2}}
$$


bezeichnet wird; genauer gesagt wird die Grösse

$$
K(\mathrm{o}, \mathrm{I})+\frac{\mathrm{I}}{2 \pi} \log \mathrm{P}_{\mathrm{or}}
$$

auch dann, wenn die Punkte o und I zusammenrücken, endlich bleiben.

Damit ist gezeigt, dass die Funktion $K(\mathrm{O}, \mathrm{I})$ im Falle $b>0$ alle Eigenschaften der Green'schen Funktion besitzt, und mit ihr nach deren Definition identisch ist. Im Falle $b=0$ hat man die erweiterte GreEn'sche Funktion, die zuerst von STEkLofF ${ }^{6}$ ) eingeführt ist, und zu ähnlichen Zwecken wie die gewöhnliche gebraucht werden kann, falls diese nicht existiert. Der explizite Ausdruck der GreEs'schen Funktion scheint bisher nur im Falle $b=\infty$ bekannt gewesen zu sein.

Aus den angeführten Eigenschaften der Green'schen Funktion und den Differentialgleichungen

$$
\Delta \varphi_{m \mu}+k_{m \mu}^{2} \varphi_{m \mu}=0, \quad \Delta \bar{\varphi}_{m \mu}+k_{m \mu}^{2} \bar{\rho}_{m \mu}=0
$$

ergeben sich leicht durch die Gauss'sche Integraltransformation die Formeln

$$
\varphi_{m \mu} \mathrm{I}=k_{m \mu}^{2} \int_{G} K(\mathrm{o}, \mathrm{I}) \varphi_{m \mu} \text { o.d } s_{\mathrm{o}}, \quad \bar{\varphi}_{m \mu} \mathrm{I}=k_{m \mu}^{2} \int_{G} K(\mathrm{o}, \mathrm{I}) \bar{\varphi}_{m \mu} \mathrm{o} . d s_{\mathrm{o}},
$$

die für den Fall $b=\infty$ schon vor der allgemeinen Theorie der Integralgleichungen bekannt waren ${ }^{7}$ ).

\section{$\$ 3$.}

\section{Entwicklung einer Funktion mit unstetigen Ableitungen nach den Eigenfunktionen.}

Es sei $L$ eine sich selbst nicht schneidende Kurve, die sich entweder im Innern der Kreisfläche $G$ schliesst, oder zwei Punkte der Randlinie $r=$ I verbindet; $d l$ sei ihr Bogenelement und $F$ eine Funktion des Ortes auf ihr, die auf der Randlinie, wenn die Kurve $L$ an diese heranreicht, verschwindet. Als Funktionen der Bogenlänge $l$ mögen $F$, die Polarkoordinaten $r, \theta$ und die Ableitungen dieser Grössen solche Stetigkeitseigenschaften besitzen, dass, wenn man $F$ als Dichtigkeit einer auf der Linie $L$ verteilten Masse ansieht, die gewöhnlichen Eigenschaften des logarithmischen Potentials dieser Masse erhalten bleiben; dass ferner jeder der Ausdrücke

$$
F \frac{d \theta}{d r}, \quad F \frac{d r}{d \theta}
$$

auf einer Strecke, auf der er endlich bleibt, stets von beschränkter Schwankung ist, d. h. als Differenz monotoner Funktionen gleichen Vorzeichens dargestellt werden kann. $\mathrm{Zu}$ diesem $Z$ weck genügt es anzunehmen, dass $F, r, \theta$ stetige Ableitungen erster

6) Vergl. S. 247 der in Anm. ${ }^{x}$ ) zitierten Abhandlung.

7) Schwarz, Gesammelte Abhandlungen, Bd. I, S. 246. 
und zweiter Ordnung nach $l$ besitzen, dass die Grössen $\frac{d r}{d l}, r \frac{d \theta}{d l}$ nicht zugleich vèrschwinden, und dass jede von ihnen sowie die Grösse $r$ längs der Kurve $L$ einen beliebig festgesetzten Wert nur eine endliche Anzahl von Malen erreicht, also nicht unendlich oft vom Wachsen zum Abnehmen übergeht oder umgekehrt.

Dies festgesetzt bilden wir die Grösse

$$
P=P_{\mathrm{I}}=\int_{L} F \mathrm{o} . K(\mathrm{o}, \mathrm{r}) d l \text {. }
$$

Da $K(\mathrm{O}, \mathrm{I})$ sich nach $\ 2$ von der Grösse $\frac{\mathrm{I}}{2 \pi} \log \frac{\mathrm{I}}{\mathrm{P}_{\mathrm{or}}}$ nur um einen in der ganzen Fläche $G$ stetigen Ausdruck, der von den beiden Stellen o und I abhängt, unterscheidet, so hat die Grösse $P$, als Funktion der Stelle I betrachtet, im wesentlichen den Charakter eines logarithmischen Potentials einer auf der Linie $L$ verteilten Masse von der Dichtigkeit $F_{0}: 2 \pi$. Die Grösse $P_{I}$ ist daher eine auf der ganzen Kreisfläche $G$ mit Einschluss des Randes stetige Funktion des Ortes I. Auch wenn die Linie $L$ Randpunkte verbindet, bleibt $P$ in der Nähe derselben und in ihnen endlich und stetig, da die Dichtigkeit der Belegung in diesen Punkten nach Voraussetzung verschwindet. Die Differentialquotienten der Grösse $P$ sind auf dem ganzen Gebiet $G$ endlich und mit Ausnahme der Linie $L$ stetig; in dieser selbst besteht, wenn ihre Normalen durch $n$ und $n^{\prime}$ bezeichnet werden, die Gleichung

$$
\frac{\partial P_{\mathrm{I}}}{\partial n_{\mathrm{I}}}+\frac{\partial P_{\mathrm{I}}}{\partial n_{\mathrm{I}}^{\prime}}=-F_{\mathrm{I}}
$$

Betreffs der hier und im Folgenden benutzten Eigenschaften des Potentials werde auf eine Abhandlung von Plemelj ${ }^{8}$ ) verwiesen.

Wir wollen nun in den Ausdruck der Grösse $P$ für $K(\mathrm{O}, \mathrm{I})$ die im $\ 2$ angegebene Reihenentwicklung einsetzen und zeigen, dass die Grösse $P$ auf die Fourier'sche Art nach den Funktionen $\varphi$ und $\bar{\varphi}$ entwickelt werden kann; damit wird ein erstes Beispiel einer entwickelbaren Funktion gegeben sein, die nicht auf der ganzen Fläche $G$ stetige Ableitungen darbietet.

Um dieses Ziel zu erreichen, gehen wir davon aus, dass die Grösse $K(\mathrm{o}, \mathrm{r})$, abgesehen von einem oder zwei zu Anfang stehenden Gliedern von besonderer Form, aus Reihen von der Form

$$
\sum_{m}^{\mathrm{r}, \infty} \frac{\alpha^{m} \cos m\left(\theta-\theta_{\mathrm{I}}\right)}{m}, \quad \sum_{m}^{\mathrm{r}, \infty} \frac{\alpha^{m} \cos m\left(\theta-\theta_{1}\right)}{m+b}
$$

zusammengesetzt ist, in denen für a einer der Werte $u$ und $r r_{x}$ zu nehmen ist, sodass $\alpha$ niemals den Wert I überschreitet. Diese Reihen können mit $F_{0}$ multipliziert längs der Linie $L$ gliedweise integriert werden, wie wir zeigen wollen.

Es sei zunächst 23 ein Bogen der Kurve $L$, längs dessen eine der Grössen

8) Über lineare Randwertaufgaben der Potentialtheorie [Monatshefte für Mathematik und Physik, Bd. XV (1904), S. 337-412], S. 346 ff. 
$\frac{d r}{d l}, \frac{d \theta}{d l}$, etwa die zweite, von Null verschieden ist und beide Grössen $r, \theta$ monoton sind. Ausserdem erreiche $r$ den Wert $r_{1}$ entweder garnicht oder nur in einem der Punkte 2 und 3 , sodass auch die Grösse $u$ ihre Gestalt behält und monoton ist; nach Voraussetzung zerfällt $L$ in eine endliche Anzahl solcher Bögen. Dann kann man setzen

$$
\begin{aligned}
& G \circ=F_{0}: \frac{d \theta}{d l}, \\
& \int_{2}^{3} F_{0} \alpha^{m} \cos m\left(\theta-\theta_{\mathrm{i}}\right) d l=\int_{2}^{3} G_{0} \cdot \alpha^{m} \cos m\left(\theta-\theta_{\mathrm{I}}\right) d \theta
\end{aligned}
$$

und die Grösse Go ist längs des Bogens 23 endlich und stetig nebst ihrer Ableitung längs dieser Kurve. Die partielle Integration ergibt nun

$$
\begin{aligned}
\int_{2}^{3} G 0 . \alpha^{m} \cos m\left(\theta-\theta_{1}\right) d \theta=\left.G o \cdot \frac{\alpha^{m} \sin \left(\theta-\theta_{1}\right)}{m}\right|_{2} ^{3} & -\int_{2}^{3} \frac{d G o}{d \theta} \alpha^{m} \frac{\sin m\left(\theta-\theta_{1}\right)}{m} d \theta \\
& -\int_{2}^{j} G 0 . \alpha^{m-1} \frac{d \alpha}{d \theta} \sin m\left(\theta-\theta_{1}\right) d \theta .
\end{aligned}
$$

Man kann ferner nach den geltenden Voraussetzungen eine Gleichung

$$
G \circ \cdot \frac{d \alpha}{d \theta}=f^{\theta}-g \theta,
$$

ansetzen, in $\operatorname{der} f$ und $g$ positive monotone Funktionen, von $\theta$ bedeuten, entweder beide wachsend oder beide abnehmend. Man verwirklicht von diesen beiden Möglichkeiten diejenige, die bewirkt, dass $f \theta$ und $\alpha$ als Funktionen von $\theta$ auf der Strecke 23 denselben Sinn des Wachsens oder Abnehmens haben. Dann gilt dasselbe auch für die Produkte

$$
\alpha^{m} f \theta, \quad \alpha^{m} g \theta ;
$$

und die Funktionen $f, g$ sind von $m$ offenbar unabhängig.

Nun gilt, wenn $\psi \theta$ auf der Strecke von $\theta_{2}$ bis $\theta_{3}$ monoton ist, allgemein die Formel ${ }^{9}$ )

$$
\int_{2}^{3} \psi \theta \cdot \sin m \theta d \theta=\frac{\Psi}{m}
$$

wobei $|\Psi|$ kleiner ist als der achtfache Wert des grössten absoluten Betrages von $\psi \theta$ auf der Integrationsstrecke, und dieselbe Formel gilt, wenn man sin durch cos ersetzt. Hieraus folgt unmittelbar, dass man setzen kann

$$
\int_{2}^{3} F \mathrm{O} \cdot \alpha^{m} \cos m\left(\theta-\theta_{\mathrm{s}}\right) d l=\frac{\Psi}{m},
$$

wobei die Grösse $\Psi$ zwischen endlichen, von $m$ unabhängigen Grenzen liegt; diese können offenbar von den Stellen I, 2 und 3 unabhängig gewählt werden, wenn sich diese so ändern, dass der Bogen 23 die vorausgesetzten Eigenschaften behält.

Genau die zuletzt erhaltene Gleichung lässt sich aber auch ableiten, wenn längs

9). KNESER, Untersuchungen über die Darstellung willkürlicher Funktionen in der mathematischen Physik [Mathematische Annalen, Bd. LVIII (1904), S. 8I-I47], S. 94.

Rend. Circ. Matem. Palermo, t. XXVII ( ${ }^{\circ}$ sem. 1909). - Stampato il 25 settembre 1908. 
des Bogens 23 die Grösse $\frac{d r}{d l}$ von Null verschieden bleibt. Alsdann kann man setzen

$$
H_{0}=F_{0}: \frac{d r}{d l}
$$

wobei die linke Seite die für $F_{0}$ vorausgesetzten Stetigkeitseigenschaften ebenfalls besitzt und man erhält die Gleichung

$$
\int_{2}^{3} F \text { o. } x^{m} \cos m\left(\theta-\theta_{1}\right) d l=\int_{2}^{3} H o \cdot \alpha^{m} \cos m\left(\theta-\theta_{1}\right) d r .
$$

Es sei z. B. $\alpha=\frac{r}{r_{1}}$; dann findet man, indem man partiell integriert,

$$
\begin{gathered}
\int_{2}^{3} H \mathrm{o} \cdot\left(\frac{r}{r_{\mathrm{I}}}\right)^{m} \cos m\left(\theta-\theta_{\mathrm{I}}\right) d r=\left.H \mathrm{o} \cdot \frac{\cos m\left(\theta-\theta_{\mathrm{s}}\right)}{m+\mathrm{I}} \frac{r^{m+\mathrm{I}}}{r_{\mathrm{I}}^{m}}\right|_{2} ^{3} \\
-\int_{2}^{3} \frac{H_{\mathrm{o}}}{d r} \cdot \frac{\cos m\left(\theta-\theta_{\mathrm{I}}\right)}{m+\mathrm{I}} \frac{r^{m+\mathrm{I}}}{r_{\mathrm{I}}^{m}} d r-\frac{m}{m+\mathrm{I}} \int_{2}^{3} H \mathrm{o} \cdot \sin m\left(\theta-\theta_{\mathrm{s}}\right) \frac{d \theta}{d r} r_{\mathrm{I}} \alpha^{m+\mathrm{I}} d r,
\end{gathered}
$$

und da die Grösse $\alpha^{m+1}$ monoton ist, kann man hier genau wie oben zeigen, dass das letzte Integral die Gestalt $\frac{\Psi}{m}$ hat, was von den ersten beiden Gliedern unmittelbar ersichtlich ist. Damit ist die Gleichung

$$
\int_{2}^{3} F_{0} \cdot \alpha^{m} \cos m\left(\theta-\theta_{\mathrm{r}}\right) d l=\frac{\mathbf{\Psi}}{m}
$$

auch für diesen Fall bewiesen und eine nur unwesentlich geänderte Argumentation ergibt dasselbe Resultat, auch wenn für $\alpha$ einer der Werte $r r_{1}$ und $\frac{r_{I}}{r}$ gesetzt werden muss.

Aus der erhaltenen Gleichung ergibt sich sofort, dass die Reihen

$$
\begin{aligned}
& \sum_{m}^{1, \infty} \int_{2}^{3} F_{0} \cdot \frac{\alpha^{m} \cos m\left(\theta-\theta_{\mathrm{r}}\right)}{m} d l=S_{23} \\
& \sum_{m}^{\mathrm{r}, \infty} \int_{2}^{3} F_{\mathrm{O}} \cdot \frac{\alpha^{m} \cos m\left(\theta-\theta_{\mathrm{r}}\right)}{m+b} d l=\bar{S}_{23}
\end{aligned}
$$

bei beliebiger fester Lage des Punktes I konvergieren und zwar wie die Summen

$$
\sum \frac{\Psi}{m^{2}}, \quad \sum \frac{\Psi}{m(m+b)},
$$

in denen der Buchstabe $\Psi$ die oben angegebene Bedeutung hat. Da die einzelnen Glieder dieser Reihen stetige Funktionen der Stellen I, 2 und 3 sind, gilt dasselbe auch von den Summen, solange die für den Bogen 23 aufgestellten Voraussetzungen gültig bleiben.

Halten wir zunächst die Punkte 2 und 3 fest und lassen I variieren. Dann hört die vom Bogen 23 geforderte Eigenschaft nur auf, wenn $r_{\mathrm{x}}$ einen der Werte $r_{2}$ oder $r_{3}$ überschreitet, sodass der Wert $r_{\mathbf{x}}$ im Innern der Strecke 23, etwa im Punkte 4 erreicht wird. Man braucht aber die Strecke 23 nur in die beiden Teile 24 und 43 zu teilen, die alle vorher von 23 geforderten Eigenschaften besitzen. Die Summen $S_{24}$, 
$\bar{S}_{24}, S_{43}$ und $\bar{S}_{43}$ sind dann, auch wenn der Punkt 4 in die Lage 2 oder 3 übergeht, stetige Funktionen der Stelle I; mithin gilt dasselbe von der Summe $S_{23}$ auch, wenn die hinsichtlich der Stelle I gemachte Voraussetzung für den Bogen 23 nicht mehr gilt, sodass jetzt der Strecke 23 nur noch Eigenschaften auferlegt werden, die von der Kurve $L$ allein abhängen. Hiermit ist gezeigt, dass die Summen $S_{23}$ und $\bar{S}_{23}$ unter unseren Voraussetzungen stetige Funktionen der Stelle I auf dem ganzen Gebiet $G$ sind, und da die Kurve $L$ in eine endliche Anzahl solcher Strecken zerfällt, gilt dasselbe von den Summen $S_{L}, \bar{S}_{L}$, die aus $S_{23}, \bar{S}_{23}$ hervorgehen, wenn man als Integrationsstrecke überall die ganze Kurve $L$ benutzt.

Jetzt wird es leicht, die Grösse $P$ in der erwünschten Form zu entwickeln.

Setzen wir nämlich

$$
P_{23}=\int_{2}^{3} F \mathrm{o} . K(\mathrm{o}, \mathrm{I}) d l
$$

und betrachten zunächst wieder einen Bogen 23, auf dem der Wert $r_{1}$ nicht erreicht wird, so konvergieren die Reihen

$$
\sum_{m}^{\mathrm{r}, \infty} \frac{\alpha^{m} \cos m\left(\theta-\theta_{\mathrm{r}}\right)}{m}, \quad \sum_{m}^{\mathrm{r}, \infty} \frac{\alpha^{m} \cos m\left(\theta-\theta_{\mathrm{r}}\right)}{m+b}
$$

auf diesem Bogen, da $\propto$ unter einer von I verschiedenen Grenze bleibt, gleichmässig und können gliedweise integriert werden. Daraus ergibt sich, dass man $P_{23}$ erhält, indem man die Reihenentwicklung $K(\mathrm{o}, \mathrm{I})$ in den Integralausdruck einsetzt und gliedweise integriert. Die so erhaltene Summe von unendlich vielen Integralen setzt sich aus drei Summen $S_{2 j}, \bar{S}_{2 j}$ und höchstens zwei einzelnen Gliedern zusammen. Bezeichnen wir daher die durch Integration der einzelnen Summanden entstandenen Summen durch $T_{23}$, so ist die Gleichung

$$
P_{23}=T_{23}
$$

bewiesen unter der Voraussetzung, dass $r_{\mathrm{r}}$ auf der Strecke 23 nicht erreicht wird, und die Grösse $T_{23}$ ist ebenso wie die einzelnen in ihr vorkommenden Grössen $S_{23}, \bar{S}_{23}$ eine stetige Funktion der Stellen I, 2, 3; dabei kann die Stelle I auf der ganzen Kreisfläche $G$ beliebig wandern.

Jetzt lasse man den Punkt 3 gegen eine Stelle 4 heranrücken, in der $r_{4}=r_{1}$ ist; dann folgt aus der Stetigkeit beider Seiten der Gleichung

dass auch die Gleichung

$$
P_{23}=T_{23}
$$

$$
P_{24}=T_{24}
$$

gilt. Da nun nach dem früheren die Kurve $L$ in eine endliche Anzahl von Bögen 23 zerlegt werden kann, auf denen der Wert $r_{1}$ höchstens in den Endpunkten erreicht wird, so folgt, indem man eine endliche Anzahl von Gleichungen von der Form der zuletzt erhaltenen addiert, das Resultat

$$
P_{L}=T_{L},
$$

oder die Grösse $P$ kann in der Tat in der Weise wie es behauptet wurde, indem man 
gliedweise integriert, in eine Reihe entwickelt werden, für die man auch in der Bezeichnung des $\ 2$ schreiben kann

$$
P=\int_{L} F \mathrm{o} . K(\mathrm{o}, \mathrm{I}) d l=\frac{\mathrm{I}}{\pi} \sum_{m}^{0, \infty} \int_{L} F \mathrm{o} . K\left(r_{\mathrm{o}}, r_{\mathrm{r}}\right)_{m} \cos m\left(\theta-\theta_{\mathrm{s}}\right) d l .
$$

Jedes einzelne Glied der rechten Seite hat nun die Form

$$
\int_{L} K\left(r, r_{\mathrm{r}}\right)_{m} G \text { o.dl }
$$

und ein solches Integral kann nach $\ 2$ gliedweise integriert werden. Denn nach unseren Voraussetzungen ändert das Differential $d r$ sein Vorzeichen nur eine endliche Anzahl von Malen; die Voraussetzungen der zitierten Stelle sind also erfüllt. Setzt man für $K\left(r_{\mathrm{o}}, r_{\mathrm{s}}\right)_{m}$ nach $\ 2$ die Reihen ein und verfährt in der angegebenen Weise, so findet man

$$
\begin{aligned}
& \int_{L} F \text { o. } K\left(r_{0}, r_{1}\right)_{n} \cos m\left(\theta-\theta_{1}\right) d l \\
& =\sum_{\mu}^{\mathrm{r}, \infty}\left[\frac{J_{m}\left(k_{m \mu} r_{\mathrm{I}}\right) \cos m \theta_{\mathrm{I}}}{\pi k_{m \mu}^{2} N_{m \mu}} \int_{L} F \mathrm{o} . J_{m}\left(k_{m \mu} r\right) \cos m \theta \cdot d l\right. \\
& \left.+\frac{J_{m}\left(k_{m \mu} r_{1}\right) \sin m \theta_{1}}{\pi k_{m \mu}^{2} N_{m \mu}} \int_{L} F \text { o. } J_{m}\left(k_{m \mu} r\right) \sin m \theta . d l\right] \\
& =\sum_{\mu}^{\mathrm{r}, \infty}\left[\frac{\varphi_{m \mu} \mathrm{I}}{\lambda_{m \mu}} \int_{L} F_{0} . \varphi_{m \mu} \mathrm{o} . d l+\frac{\bar{\varphi}_{m \mu} \mathrm{I}}{\lambda_{m \mu}} \int_{L} F_{\mathrm{o}} . \bar{\varphi}_{m \mu} \mathrm{o} . d l\right] .
\end{aligned}
$$

Nun ergibt die Integralgleichung, die die Funktionen $\varphi, \bar{\varphi}$ nach $\ 2$ erfüllen, die Integraltransformation

$$
\begin{aligned}
\int_{L} \frac{\varphi_{m \mu} \mathrm{o}}{\lambda_{m \mu}} F \mathrm{o} . d l & =\int_{L} F \mathrm{o} . d l_{0} \int_{G} K(\mathrm{o}, 2) \varphi_{m \mu} 2 . d s_{2} \\
& =\int_{G} P_{0} . \varphi_{m \mu} \mathrm{o} . d s,
\end{aligned}
$$

in der auch $\varphi$ durch $\bar{\varphi}$ ersetzt werden kann; kombiniert man sie mit der vorletzten Gleichung, so ist die Grösse $P$ genau auf die Fourier'sche Weise in eine Reihe entwickelt, deren Glieder allerdings in einer besonderen Reihenfolge angeordnet sind. Setzen wir

so ist die Gleichung

$$
A_{m \mu}=\int_{G} P o . \varphi_{m \mu} \text { o.ds, } \bar{A}_{m \mu}=\int_{G} P o . \bar{\varphi}_{m \mu} \text { o.ds }
$$

bewiesen.

$$
P \mathrm{o}=\sum_{m}^{0, \infty} \sum_{\mu}^{1, \infty}\left[A_{m \mu} \varphi_{m \mu} \mathrm{o}+\bar{A}_{m \mu} \bar{\varphi}_{m \mu} \mathrm{o}\right]
$$

Es ist also in der Tat, wie wir es anstrebten, eine Funktion, deren Ableitungen gewisse ziemlich allgemeine Unstetigkeiten darbieten dürfen, auf die FourIER'sche Art nach den Eigenfunktionen unserer Probleme entwickelt. 


\section{$\$ 4$.}

\section{Entwicklung einer unstetigen Funktion nach den Eigenfunktionen.}

Die Symbole $F, L, n$ mögen ihre Bedeutung behalten; mit ihnen bilden wir jetzt den Ausdruck

$$
Q=\int_{L} F_{\mathrm{o}} \cdot \frac{d K(\mathrm{o}, \mathrm{I})}{d n_{\mathrm{o}}} d l_{\mathrm{o}}
$$

Ersetzen wir in ihm die ganze Linie $L$ durch den Bogen 23, so gehe er in $Q_{23}$ über und analoge Bedeutung habe die Grösse $Q$ mit zwei Zeigern überhaupt.

Bezieht man dann die Differentiale $d r$ und $d \theta$ auf diejenige Fortgangsrichtung längs der Kurve $L$, die zur Normalen $n$ so liegt wie die Richtung wachsender $r$ zur Richtung wachsender $\theta$ längs eines Kreises $r=$ const., so findet man leicht die Identität

$$
d l \frac{d}{d n}=-r \frac{\partial}{\partial r} \cdot d \theta+\frac{\mathrm{I}}{r} \frac{\partial}{\partial \theta} \cdot d r .
$$

Führt man in diese Formel die Grösse $u$ ein, die ebenso wie früher dem echt gebrochenen der beiden Werte $\frac{r}{r_{\mathrm{I}}}$ und $\frac{r_{\mathrm{I}}}{r}$ gleich sei, so findet man

$$
d l \frac{d}{d n}=\varepsilon\left\{-u \frac{\partial}{\partial u} \cdot d \theta+\frac{\mathrm{I}}{u} \frac{\partial}{\partial \theta} \cdot d u\right\},
$$

wobei $\varepsilon$ die Einheit mit dem Vorzeichen der Grösse $\log \frac{r_{1}}{r}$ bedeutet. Hieraus findet man weiter

$$
d l \frac{d}{d n}\left[u^{m} \cos m\left(\theta-\theta_{\mathrm{t}}\right)\right]=-\varepsilon d\left[u^{m} \sin m\left(\theta-\theta_{\mathrm{I}}\right)\right] .
$$

Ferner ergibt sich, wenn $v=r r_{\mathrm{I}}$ gesetzt wird,

$$
d l \frac{d}{d n}\left[v^{m} \cos m\left(\theta-\theta_{1}\right)\right]=-d\left[v^{m} \sin m\left(\theta-\theta_{\mathrm{r}}\right)\right] \text {. }
$$

Bei der festgesetzten Bedeutung der Grössen $d r, d \theta$ sind diese Formeln nur so anzuwenden, dass man eine bestimmte Integrationsrichtung festhält, die bei der gewöhnlichen Orientierung des Koordinatensystems dadurch definiert werden kann, dass für eine in dieser Richtung wandernde Figur die Richtung $n$ nach links weist.

I. Wenn nun zunächst $Q_{23}$ gebildet wird und der Wert $r_{5}$ auf dem Bogen 23 nicht erreicht wird, so bleiben die Grössen $u$ und $v$ sicher unterhalb eines festen echten Bruches, und die Reihen, aus denen sich, abgesehen von zwei Gliedern, die bei Konvergenzfragen nicht berücksichtigt $\mathrm{zu}$ werden brauchen, $K(\mathrm{O}, \mathrm{I})$ zusammensetzt, nämlich

$$
\begin{gathered}
U=\sum_{m}^{\mathrm{r}, \infty} u^{m} \frac{\cos m\left(\theta-\theta_{\mathrm{s}}\right)}{2 \pi m}, \quad V=\sum_{m}^{1, \infty} v^{m} \frac{\cos m\left(\theta-\theta_{1}\right)}{2 \pi m}, \\
W=\sum_{m}^{\mathrm{I}, \infty} v^{m} \frac{\cos m\left(\theta-\theta_{\mathrm{I}}\right)}{2 \pi m(m+b)}
\end{gathered}
$$


konvergieren gleichmässig. Sie sind ferner die reellen Teile leicht angebbarer Potenzreihen der Argumente $r e^{\theta i}$ oder $r^{-1} e^{-\theta i}$, können also nach $r$ und $\theta$ gliedweise differenziert und die so erhaltenen Reihen wiederum gliedweise, nachdem sie mit stetigen Faktoren multipliziert sind, integriert werden. Mithin kann man auch die Operation $d l \frac{d}{d n}$ in dem Ausdruck $Q_{23}$, indem man für $K(\mathrm{o}, \mathrm{x})$ die Reihe

$$
\frac{\mathrm{I}}{2 \pi b}-\frac{\mathrm{I}}{2 \pi} \log \frac{r r_{\mathrm{I}}}{u}+U+V+W
$$

einsetzt, gliedweise ausführen und dann gliedweise integrieren, womit man eine Reihenentwickelung der Grösse $Q_{23}$ erhält. Diese Reihe setzt sich aus Gliedern zusammen, die von den Reihen $U, V, W$ herrühren; setzt man allgemein

$$
\begin{aligned}
A_{23}=\int_{2}^{3} F \circ \cdot \frac{d U}{d n} d l, \quad B_{23}=\int_{2}^{3} F \circ \cdot \frac{d V}{d n} d l, \quad C_{23}=\int_{2}^{3} F \circ \cdot \frac{d W}{d n} d l, \\
A_{23}^{\prime}=\varepsilon \sum_{m}^{1, \infty} \int_{2}^{3} \frac{F_{0}}{2 \pi m} d\left(u^{m} \sin m\left(\theta-\theta_{\mathrm{r}}\right)\right), \\
B_{23}^{\prime}=-\sum_{m}^{\mathrm{r}, \infty} \int_{2}^{3} \frac{F_{\mathrm{o}}}{2 \pi m} d\left(v^{m} \sin m\left(\theta-\theta_{\mathrm{r}}\right)\right), \\
C_{23}^{\prime}=-\sum_{m}^{\mathrm{r}, \infty} \int_{2}^{3} \frac{F_{\mathrm{O}}}{2 \pi m(m+b)} d\left(w^{m} \sin m\left(\theta-\theta_{1}\right)\right),
\end{aligned}
$$

so findet man aus den soeben erhaltenen Formeln

$$
A_{23}=A_{23}^{\prime}, \quad B_{23}=B_{23}^{\prime}, \quad C_{23}=C_{23}^{\prime} \text {. }
$$

II. Zweitens betrachten wir einen Bogen 24 der Kurve $L$, auf welchem $r_{4}=r_{1}$, $\theta_{4}$ aber von $\theta_{1}$ verschieden ist, sodass die Stellen 4 und I nicht zusammenfallen; liegt 3 zwischen 2 und 4 , so habe der Bogen 23 stets die unter I. festgehaltenen Eigenschaften, d. h. 4 sei von 2 aus in einer gewissen Richtung auf der Kurve $L$ der erste Punkt, in welchem $r$ den Wert $r_{1}$ erreicht.

Dann wollen wir zeigen, dass die Grössen $A_{23}, B_{23}, C_{23}$ ebenso wie die ihnen gleich gefundenen Reihen $A_{23}^{\prime}, B_{23}^{\prime}, C_{23}^{\prime}$ auf der ganzen Strecke 24 stetige Funktionen der Stelle 3 sind. Daraus ergeben sich dann nach der gewöhnlichen Schlussweise der Exhaustionsmethode die Gleichungen

$$
A_{24}=A_{24}^{\prime}, \quad B_{24}=B_{24}^{\prime}, \quad C_{24}=C_{24}^{\prime} .
$$

Zunächst erweisen sich die Grössen $A, B, C$ leicht als stetig. Denn die Grösse $C_{23}$ macht keine Schwierigkeit, da die Ableitungen der Grösse $W$ auf der Fläche $G$ überall endlich und stetig sind, solange nicht die Gleichungen

gelten.

$$
v=\mathrm{r}, \quad r=r_{\mathrm{r}}=\mathrm{x}, \quad \theta=\theta_{\mathrm{x}}
$$

Sodann sieht man auf Grund der Formel

$$
-\sum_{m}^{1, \infty} \frac{\alpha^{m} \cos m \beta}{m}=\log \sqrt{\mathrm{I}-2 \alpha \cos \beta+\alpha^{2}},
$$

die auch bei der Annahme $\alpha=$ I gilt, unmittelbar, dass die Grössen $A$ und $B$ im 
wesentlichen logarithmische Potentiale von Doppellinien sind, d. h. auf der Fläche $G$ stetige Funktionen von I, 2 und 3 sind, solange die Stelle I, wie wir ja voraussetzen, nicht auf dem doppelt belegten Bogen 23 liegt.

Die Reihen $A_{23}^{\prime}, B_{23}^{\prime}, C_{23}^{\prime}$ erfordern eine etwas längere Betrachtung. Offenbar gilt die Gleichung

$$
\int_{2}^{j} F_{0 . d}\left[\frac{\sin m\left(\theta-\theta_{1}\right)}{2 \pi m} u^{m}\right]=\left.\frac{F_{0} \cdot u^{m} \sin m\left(\theta-\theta_{\mathrm{r}}\right)}{2 \pi m}\right|_{2} ^{3}-\int_{2}^{3} d F_{\mathrm{o}} \cdot \frac{u^{m} \sin m\left(\theta-\theta_{1}\right)}{2 \pi m} ;
$$

summiert man also nach $m$ und berücksichtigt die Formel

$$
\operatorname{arctg} \frac{\alpha \sin \beta}{\mathrm{I}-\alpha \cos \beta}=\sum_{m}^{\mathrm{I}, \infty} \frac{\alpha^{m} \sin m \beta}{m},
$$

die auch gilt, wenn $\alpha=\mathrm{I}$ und $\sin \beta$ von Null verschieden ist, so folgt

$$
A_{23}^{\prime}=\left.\frac{F_{0}}{2 \pi} \operatorname{arctg} \frac{u \sin \left(\theta-\theta_{1}\right)}{\mathrm{I}-u \cos \left(\theta-\theta_{\mathrm{s}}\right)}\right|_{2} ^{3}-\sum_{m}^{\mathrm{r}, \infty} \int_{2}^{3} d F_{\mathrm{o}} . \frac{u^{m} \sin m\left(\theta-\theta_{1}\right)}{2 \pi m} .
$$

Hier ist zunächst die an zweiter Stelle stehende Summe bei beliebiger Aenderung der Stelle 3, auch wenn diese in die Stelle 4 hineinrückt, gleichmässig konvergent, also stetig; denn nach $\$ 3$ kann man

$$
\int_{2}^{3} d F_{0} \cdot u^{m} \sin m\left(\theta-\theta_{1}\right)=\frac{\Psi}{m}
$$

setzen, wrobei $\Psi$ zwischen von $m$ und der Stelle 3 unabhängigen endlichen Grenzen liegt. Die bezeichnete Summe konvergiert also besser als die mit einer passenden Konstanten multiplizierte Reihe

Was aber die Grösse

$$
\sum \frac{1}{m^{2}}
$$

$$
\operatorname{arctg} \frac{u \sin \left(\theta-\theta_{\mathrm{r}}\right)}{\mathrm{I}-u \cos \left(\theta-\theta_{\mathrm{s}}\right)}
$$

betrifft, so nähert sie sich, wenn man $u$ und $\theta$ in $u_{4}$ oder $u_{i}$ und $\theta_{4}$ übergehen lässt, stetig der endlichen Grenze

$$
\begin{aligned}
\operatorname{arctg} \frac{\sin \left(\theta_{4}-\theta_{1}\right)}{1-\cos \left(\theta_{4}-\theta_{1}\right)} & =\operatorname{arctg} \operatorname{cotg} \frac{\theta_{4}-\theta_{1}}{2} \\
& =\frac{\pi}{2}-\frac{\theta_{4}-\theta_{1}}{2}
\end{aligned}
$$

an, was aber wesentlich darauf beruht, dass $\theta_{4}-\theta_{1}$ von Null verschieden ist. Damit ist gezeigt, dass in der Tat die Grösse $A^{\prime}$ die behauptete Stetigkeitseigenschaft besitzt und dass die Gleichung $A_{24}=A_{24}^{\prime}$ gilt.

Genau die gleiche Schlussweise gilt für die Grösse $B^{\prime}$, während die Stetigkeit der Reihe $C^{\prime}$, wenn man das einzelne Integral durch partielle Integration umformt, unmittelbar ersichtlich ist.

Liegt der Punkt I nicht auf der Kurve $L$, so kann man diese den geltenden Voraussetzungen zufolge in eine endliche Anzahl solcher Bögen wie 24 zerlegen und 
erhält so das Resultat, dass in der Tat in dem Ausdruck

$$
Q=\int_{L} F \text { o. } \frac{d K(\mathrm{o}, \mathrm{I})}{d n_{\mathrm{o}}} d l_{\mathrm{o}},
$$

wenn man für $K(\mathrm{o}, \mathrm{r})$ die die angegebenen Reihen einsetzt, gliedweise differenziert und integriert werden kann. Dasselbe gilt offenbar, wenn der Punkt I auf der Kurve $L$ liegt, die Integration aber über einen Teil der Kurve erstreckt wird, der den Punkt I nicht enthält.

III. Wir nehmen drittens an, der Punkt I liege auf der Kurve $L$ und sei mit dem bisher schon betrachteten Punkte 4 identisch; dabei sei $r_{1}<\mathrm{I}$, der Punkt $\mathrm{I}$ also nicht auf dem Rande des Gebiets $G$. gelegen. Die Punkte 2, 3, I, 5, 6 mögen in dieser Folge längs der Kurve $L$ angeordnet sein, und der Wert $r_{\text {s }}$ werde zwischen 2 und 3 sowie zwischen 5 und 6 nicht wieder erreicht, sodass auf diesen Strecken $\varepsilon$ jeweils einen konstanten Wert und $u$ eine feste Form hat; auf der Strecke 56 bezeichnen wir diese Grössen durch $\bar{\varepsilon}$ und $\bar{u}$. Dann zeigt nur die Summe

$$
T \mathrm{o}=\sum_{m}^{1, \infty} \frac{F_{\mathrm{O}}}{2 \pi m} u^{m} \sin m\left(\theta-\theta_{\mathrm{1}}\right)
$$

ein anderes Verhalten als bisher; denn offenbar hat man die Gleichung

$$
T_{4}=\mathrm{o}
$$

während, wie man leicht sieht, $\lim T_{3}$, wenn man die Stelle 3 in die Lage 4 übergehen lässt, im allgemeinen von Null verschieden ist. In der Tat, wird in der Nähe der Stelle I auf dem Bogen 2I gesetzt

auf der Strecke I6 dagegen

$$
u_{3}=\mathrm{I}+n, \quad \theta_{3}-\theta_{1}=\omega
$$

so hat man die Gleichung

$$
\bar{u}_{s}=\mathrm{I}+\bar{n}, \theta_{s}-\theta_{\mathrm{r}}=\bar{\omega},
$$

$$
T_{3}=\frac{F_{3}}{2 \pi} \operatorname{arctg} \frac{(\mathrm{I}+n) \sin \omega}{\mathrm{I}-(\mathrm{I}+n) \cos \omega}
$$

also wenn $n$ und $\omega$ unendlich abnehmen, und zunächst an der Stelle I kein Extremum von $r$ liegt,

$$
\begin{gathered}
\lim T_{3}=\frac{F_{\mathrm{I}}}{2 \pi} \operatorname{arctg} \frac{\omega}{-n} \\
=-\left.\frac{F_{\mathrm{I}}}{2 \pi} \operatorname{arctg} \frac{d \theta}{d u}\right|^{1}=s, \\
\lim T_{3}=T_{4}+s .
\end{gathered}
$$

In der Reihe $A_{2 \mathfrak{j}}^{\prime}$, die wir für die Grösse

$$
A_{23}=\int_{2}^{3} F \text { o. } \frac{d U}{d n} d l
$$

gefunden haben, ist nun, wenn der Punkt 3 in der festgesetzten Weise variiert, ein Bestandteil $\varepsilon T_{j}$, der allein unstetig ist, wenn der Punkt 3 die ganze Strecke 24 mit Ein- 
schluss der Grenzen durchläuft; man hat daher auf Grund des letzten Resultats

$$
\begin{aligned}
& \lim \sum_{m}^{\mathrm{r}, \infty} \int_{2}^{3} F \text { o. } \frac{d}{d n}\left[\frac{u^{m} \cos m\left(\theta-\theta_{1}\right)}{2 \pi m}\right] d l \\
= & \varepsilon s+\sum_{m}^{1, \infty} \int_{2}^{4} F_{0} \cdot \frac{d}{d n}\left[\frac{u^{m} \cos m\left(\theta-\theta_{1}\right)}{2 \pi m}\right] d l
\end{aligned}
$$

wobei das Zeichen $\lim$ wie vorher $\mathrm{zu}$ verstehen ist.

Genau auf dieselbe Art findet man, da die Grösse $\int_{s}^{6} F$ o. $\frac{d U}{d n} d l$ als unstetigen Bestandteil den Summanden $-\bar{\varepsilon} T S$ enthält, wenn man den Punkt 5 an die Lage I heranrücken lässt,

$$
\begin{gathered}
\lim \sum_{m}^{\mathrm{r}, \infty} \int_{5}^{6} F \text { o. } \frac{d}{d n}\left[\frac{\bar{u}^{m} \cos m\left(\theta-\theta_{\mathrm{I}}\right)}{2 \pi m}\right] d l \\
=-\bar{\varepsilon} \bar{s}+\sum_{m}^{\mathrm{r}, \infty} \int_{\mathrm{I}}^{6} F \text { o. } \frac{d}{d n}\left[\frac{\bar{u}^{m} \cos m\left(\theta-\theta_{\mathrm{s}}\right)}{2 \pi m}\right] d l
\end{gathered}
$$

dabei ist

$$
\bar{s}=-\frac{F_{\mathrm{I}}}{2 \pi} \operatorname{arctg} \frac{d \theta}{d \bar{u}}
$$

zu setzen.

Nun ist am Punkte I offenbar

also folgt

$$
d u=-d \vec{u}
$$

$$
\bar{s}=-s
$$

und da $\varepsilon$ und $\bar{\varepsilon}$ entgegengesetzt sind, unter der Voraussetzung, dass $r$, wenn man die Lage I passiert, den Sinn der Aenderung beibehält, so folgt

$$
\varepsilon s-\bar{\varepsilon} \bar{s}=0 \text {. }
$$

Addiert man daher die letzten beiden mit dem Zeichen lim behafteten Gleichungen und versteht dieses Zeichen so, dass die Stellen 3 und 5 unabhängig voneinander und von verschiedenen Seiten her gegen die Lage I konvergieren, so findet man

$$
\begin{aligned}
& \lim \sum_{m}^{1, \infty}\left\{\int_{2}^{3} F \text { o. } \frac{d}{d n}\right. {\left.\left[\frac{u^{m} \cos m\left(\theta-\theta_{1}\right)}{2 \pi m}\right] d l+\int_{5}^{6} F \text { o. } \frac{d}{d n}\left[\frac{\overline{u^{m}} \cos m\left(\theta-\theta_{1}\right)}{2 \pi m}\right] d l\right\} } \\
&=\sum_{m}^{1, \infty} \int_{2}^{6} F \text { o. } \frac{d}{d n}\left[\frac{u^{m} \cos m\left(\theta-\theta_{1}\right)}{2 \pi m}\right] d l .
\end{aligned}
$$

Der Grenzwert auf der linken Seite ist aber nichts anderes als

$$
\lim \left[\int_{2}^{3} F \text { o. } \frac{d U}{d n} d l+\int_{5}^{6} F \text { o. } \frac{d U}{d n} d l\right]=\int_{2}^{6} F \text { o. } \frac{d U}{d n} d l
$$

$\mathrm{da}$ dieses Integral allgemein eine stetige Funktion der Grenze ist. Somit ist auch die rechte Seite der vorletzten Gleichung diesem Integral gleich und es ist gezeigt, dass in diesem in der Tat die Reihe $U$ gliedweise differenziert und die erhaltenen Ausdrücke gliedweise integriert werden dürfen.

Rend. Circ. Matem. Palermo, t. XXVII ( $3^{\circ}$ sem. 1909$)$. - Stampato il 29 settembre 1908. 
Die Reihen $V$ und $W$ bleiben bei unseren Voraussetzungen, da $v$ unter einem festen echtèn Bruche bleibt, auf der ganzen Strecke 26 gleichmässig konvergent, können also gliedweise differenziert und integriert werden; erinnert man sich noch der Bemerkung am Schlusse des Abschnittes II., so sieht man, dass für unseren Fall der ganze Ausdruck

$$
\int_{L} F \circ \cdot \frac{d K(\mathrm{o}, \mathrm{I})}{d n_{\mathrm{o}}} d l_{\mathrm{o}}
$$

in der behaupteten Weise gliedweise differenziert und integriert werden kann.

IV. Etwas anders liegen die Verhältnisse, wenn in I ein Extremum der Grösse $r$ vorliegt. Man hat dann etwa zu setzen

$$
n=-c \omega^{2}, \bar{n}=-c \bar{\omega}^{2}
$$

wobei $c$ eine nicht negative Konstante bedeutet und man erhält

$$
\begin{aligned}
\lim T_{3} & =\frac{F_{\mathbf{I}}}{2 \pi} \lim \operatorname{arctg} \frac{\left(\mathrm{I}-c \omega^{2}\right) \omega}{\mathrm{I}-\left(\mathrm{I}-c \omega^{2}\right)\left(\mathrm{I}-\frac{\omega^{2}}{2}\right)} \\
& =\frac{F_{\mathbf{I}}}{2 \pi} \lim \operatorname{arctg} \frac{\mathbf{I}}{\left(c+\frac{1}{2}\right) \omega},
\end{aligned}
$$

also wenn der Winkel $\theta$ in der Richtung 23 I wächst und $\omega$ negativ ist,

$$
\lim T_{3}=-\frac{F_{\mathrm{I}}}{4}=s \text {. }
$$

Ebenso ergibt sich, da unter der eben eingeführten Voraussetzung $\bar{\omega}$ positiv ist,

$$
\lim T s=+\frac{F_{\mathrm{I}}}{4}=\bar{s}
$$

wenn $\theta$ in der Richtung $23 \mathrm{I}$ abnimmt, ist in beiden Gleichungen rechts das entgegengesetzte Vorzeichen zu nehmen. Man hat also auch hier die Gleichung

$$
s=-\bar{s},
$$

dabei aber ist $\varepsilon=\bar{\varepsilon}$ zu setzen und man findet

$$
\begin{gathered}
\lim \sum_{m}^{\mathrm{I}, \infty}\left\{\int_{2}^{3} F \mathrm{o} \cdot \frac{d}{d n}\left[\frac{u^{m} \cos m\left(\theta-\theta_{1}\right)}{2 \pi m}\right] d l+\int_{5}^{6} F \mathrm{O} \cdot \frac{d}{d n}\left[\frac{u^{m} \cos m\left(\theta-\theta_{1}\right)}{2 \pi m}\right] d l\right\} \\
=\sum_{m}^{1, \infty} \int_{2}^{6} F \mathrm{o} \cdot \frac{d}{d n}\left[\frac{u^{m} \cos m\left(\theta-\theta_{1}\right)}{2 \pi m}\right] d l+2 \varepsilon s .
\end{gathered}
$$

$\mathrm{Da}$ sich die linke Seite dieser Gleichung wie oben dem Werte

$$
\int_{2}^{6} F \text { o. } \frac{d U}{d n} d l
$$

annähert, so sieht man, dass hier, wenn man gliedweise differenziert und integriert, in der Reihe das Glied

$$
2 \varepsilon s= \pm \frac{\varepsilon F_{\mathrm{I}}}{2}
$$


hinzuzufügen ist, damit die Gleichung richtig werde. Da nun die Integrale, welche $V$ und $W$ enthalten, hier ebensowenig Schwierigkeiten machen wie im vorigen Falle, so sieht man, dass die Reihenentwicklung des Ausdruckes

$$
Q=\int_{L} F \text { o. } \frac{d K(\mathrm{o}, \mathrm{I})}{d n_{\mathrm{o}}} d l_{\mathrm{o}},
$$

die man durch rein formales Verfahren erhält, die Grösse $Q$ selbst nicht darstellt, sondern vermehrt oder vermindert um $\frac{1}{2} F$ I. Erinnert man sich der Unstetigkeit des logarithmischen Potentials einer Doppellinie, welche unsere Grösse $Q$ teilt, so kann man sagen : Die Reihenentwicklung gibt an einer der Kurve $L$ angehörigen Stelle $\mathrm{I}$, in der $r_{\mathbf{I}}$ ein Extremum ist, nicht die Grösse selbst, sondern den Grenzwert, dem sie an der Stelle I zustrebt, wenn man von der einen oder anderen Seite her auf die Linie $L$ losgeht.

Diese Verhältnisse gestalten sich etwas anders, wenn die Grösse $r$ längs einer Strecke, die die Stelle I umfasst, konstant ist. Dann kann in dem Ausdruck $\frac{d K(\mathrm{o}, \mathrm{I})}{d n_{\mathrm{o}}}$ für $u$ derjenige der Werte $\frac{r}{r_{\mathrm{I}}}$ und $\frac{r_{\mathrm{I}}}{r}$ genommen werden, der ein echter Bruch wird, wenn man in der Richtung der Normale $n_{\mathrm{o}}$ die Kurve verlässt. Man hat daher $\varepsilon=+$ I oder $\varepsilon=-$ I zu setzen, jenachdem die Normale $n$ längs eines der Linie $L$ angehörigen Stückes $r=$ const. nach der Richtung abnehmender oder zunehmender Werte von $r$ zeigt.

V. Der Fall $u=v=\mathrm{I}$ kann eintreten, wenn die Kurve $L$ den Kreis $r=\mathrm{I}$ erreicht; die unter III. durchgeführte Argumentation bleibt, da auch dort bis an den Wert $u=\mathrm{I}$ heranzugehen war, gültig und kann auf die Reihe $V$ angewandt werden. Die dort unstetigen Reihen $T$ haben aber in diesem Fall keine Unstetigkeiten mehr, weil sie mit dem verschwindenden Faktor $F_{I}$ multipliziert sind; wir nehmen ja an, dass die Funktion $F$ auf dem Kreise $r=\mathrm{I}$ verschwindet. Hier bleibt also die Entwicklung des Integrals $Q$ in der gewöhnlichen Form gültig.

Zusammengefasst können wir sagen: Setzen wir wie früher

$$
K(\mathrm{o}, \mathrm{I})=\frac{\mathrm{I}}{\pi} \sum_{m}^{o, \infty} K\left(r_{0}, r_{1}\right)_{m} \cos m\left(\theta-\theta_{1}\right),
$$

so finden wir die Gleichung

$$
\begin{gathered}
Q=Q_{\mathrm{I}}=\int_{L} F_{\mathrm{o}} \frac{d K(\mathrm{o}, \mathrm{I})}{d n_{\mathrm{o}}} d l_{\mathrm{o}} \\
=\frac{\mathrm{I}}{\pi} \sum_{m}^{\mathrm{o}, \infty} \int_{L} F_{\mathrm{O}} \frac{d\left(K\left(r_{\mathrm{o}}, r_{\mathrm{1}}\right)_{m} \cos m\left(\theta_{\mathrm{o}}-\theta_{\mathrm{t}}\right)\right)}{d n_{\mathrm{o}}} d l_{\mathrm{o}}
\end{gathered}
$$

für die ganze Kreisfläche $G$ mit Ausnahme derjenigen Punkte I der Linie $L$, in denen $r_{1}$ einen extremen Wert annimmt. In diesen ist rechts der Summand $\pm \frac{I}{2} F_{I}$ zu addieren, sodass dann die ungeänderte Reihe rechts nicht den Wert $Q$ I, sondern einen der Grenzwerte darstellt, denen sich $Q_{2}$ annähert, wenn man den Punkt 2 von der einen oder anderen Seite her auf die Linie $L$ und in den Punkt I hineinrücken lässt. 
Jetzt formen wir die einzelnen Glieder der erhaltenen Reihen um und benutzen das in $\$ 2$ bewiesene Resultat: Wenn man setzt

$$
K\left(r, r_{\mathrm{s}}\right)_{m}=\sum_{\mu}^{\mathrm{r}, \infty} \frac{J_{m}\left(k_{m \mu} r\right) J_{m}\left(k_{m \mu} r_{1}\right)}{k_{m \mu}^{2} N_{m \mu}}
$$

so kann man gliedweise differenzieren und integrieren, mithin auch gliedweise die Operation $d l \frac{d}{d n}$ anwenden und dann längs der Kurve $L$ integrieren. Man erhält also

$$
\begin{gathered}
\int_{L} F \mathrm{O} \cdot \frac{d}{d n}\left[K\left(r, r_{\mathrm{1}}\right)_{m} \frac{\cos m\left(\theta-\theta_{\mathrm{I}}\right)}{\pi m}\right] d l \\
=\sum_{\mu}^{\mathrm{r}, \infty} \int_{L} F \mathrm{O} \cdot \frac{d}{d n}\left[\frac{\cos m\left(\theta-\theta_{\mathrm{I}}\right)}{\pi m} \frac{J_{m}\left(k_{m \mu} r\right) J_{m}\left(k_{m \mu} r_{\mathrm{s}}\right)}{k_{m \mu}^{2} N_{m \mu}}\right] d l,
\end{gathered}
$$

also, wenn man die in $\ 2$ eingeführten Bezeichnung der normierten Eigenfunktionen $\bar{\varphi}_{m \mu}, \varphi_{m \mu}$ benutzt,

$$
\begin{aligned}
& =\sum_{\mu}^{\mathrm{I}, \infty} \int_{L} F_{\mathrm{o}} \mathrm{o} \cdot \frac{d}{d n}\left[\frac{\varphi_{m \mu} \mathrm{o} \cdot \varphi_{m \mu} \mathrm{I}+\bar{\varphi}_{m \mu} \mathrm{o} \cdot \bar{\varphi}_{m \mu} \mathrm{I}}{\lambda_{m \mu}}\right] d l \\
& =\sum_{\mu}^{\mathrm{s}, \infty}\left[\frac{\varphi_{m \mu} \mathrm{I}}{\lambda_{m \mu}} \int_{L} F_{\mathrm{o}} \cdot \frac{d \varphi_{m \mu} \mathrm{o}}{d n} d l+\frac{\bar{\varphi}_{m \mu} \mathrm{I}}{\lambda_{m \mu}} \int_{L} F \mathrm{o} \cdot \frac{d \bar{\varphi}_{m \mu} \mathrm{o}}{d n} d l\right] .
\end{aligned}
$$

Setzt man dies Resultat in die oben für $Q$ erhaltene Reihe und benutzt die leicht ersichtlichen Identitäten

$$
\begin{aligned}
\int_{L} F \mathrm{o} . \frac{d \varphi_{m \mu} \mathrm{o}}{d n} d l & =\lambda_{m \mu} \int_{L} F \mathrm{o} . d l \frac{d}{d n} \int_{G} K(\mathrm{o}, \mathrm{I}) \varphi_{m \mu} \mathrm{I} . d s_{1} \\
& =\lambda_{m \mu} \int_{G} Q \mathrm{o} . \varphi_{m \mu} \mathrm{o} . d s_{0},
\end{aligned}
$$

so nimmt die Entwicklung der Grösse $Q$ die Gestalt einer Doppelreihe an:

wobei

$$
Q \mathrm{I}=\sum_{m}^{0, \infty} \sum_{\mu}^{\mathrm{I}, \infty}\left(A_{m \mu} \varphi_{m \mu} \mathrm{I}+\bar{A}_{m \mu} \bar{\varphi}_{m \mu} \mathrm{I}\right)
$$

$$
\begin{aligned}
& A_{m \mu}=\int_{G} Q \circ . \varphi_{m \mu} \mathrm{o} . d s_{\mathrm{o}} \\
& \bar{A}_{m \mu}=\int_{G} Q \mathrm{o} \cdot \bar{\varphi}_{m \mu} \mathrm{o} . d s_{\mathrm{o}}
\end{aligned}
$$

gesetzt ist. Die erhaltene Entwicklung ist also nach der Fourier'schen Art gebildet, und man hat eine solche Entwicklung für eine Funktion erhalten, die auf der Linie $L$ unstetig ist, während ihre Ableitungen erster und zweiter Ordnung stetig bleiben. $\mathrm{Da} Q$ bis auf eine an der Linie $L$ stetig bleibende Funktion der Stelle I mit dem Potential der Doppellinie

$$
\frac{\mathrm{I}}{2 \pi} \int_{L} d l . F \mathrm{O} \cdot \frac{d}{d n} \log \frac{\mathrm{I}}{\mathrm{Por}_{\mathrm{O}}}
$$

übereinstimmt, so beträgt der Sprung der Funktion $Q$, d.h. der Unterschied zwischen dem Grenzwert, wenn man sich einer Stelle o der Linie $L$ annähert, und dem Wert 
auf dieser Stelle selbst $\frac{1}{2} F$ o. Abgesehen von den Stellen, in denen $r$ einen extremen Wert annimmt, ist also der Wert der Reihe auf der Kurve $L$ selbst das arithmetische Mittel der Werte, denen die Grösse $Q$ zustrebt, wenn man sich von der einen oder andern Seite der Kurve $L$ annähert. Einem dieser Werte selbst ist die Reihe in den bezeichneten Ausnahmestellen gleich.

$$
\int 5 \text {. }
$$

\section{Entwicklung einer der Randbedingung nicht unterworfenen Funktion nach den Eigenfunktionen.}

Unser letztes Ziel ist, eine auf die Fourier'sche Art entwickelbare Funktion zu bilden, die der für die Eigenfunktionen geltenden Randbedingung widerspricht. Zu diesem Zweck legen wit den Punkt o auf den Kreis $r=\mathrm{I}$ und bilden, indem wir den Fall $b=\infty$ zunächst ausschliessen, die Grösse

$$
R=R \mathrm{I}=\int_{r_{\mathrm{o}}=\mathrm{r}} F_{\mathrm{o}} K(\mathrm{o}, \mathrm{I}) d l_{\mathrm{o}}
$$

wobei $r_{1}<\mathrm{I}$ sei. Aus den in $\ 2$ gegebenen Formeln für $K$ folgt, da $r=\mathrm{I}, r_{1}<\mathrm{I}$, unmittelbar die Gleichung

$$
\pi K(\mathrm{o}, \mathrm{I})=\frac{\mathrm{I}}{2 b}+\sum_{m}^{\mathrm{I}, \infty} \frac{r_{\mathrm{s}}^{m} \cos m\left(\theta-\theta_{\mathrm{I}}\right)}{m+b} .
$$

Die Reihe auf der rechten Seite ist der reelle Teil einer Potenzreihe des Arguments $r_{1} e^{i\left(\theta-\theta_{1}\right)}$; man kann also gliedweise differenzieren und erhält

$$
\pi \frac{d K(\mathrm{o}, \mathrm{I})}{d r_{\mathrm{I}}}=\sum_{m}^{\mathrm{r}, \infty} \frac{m r_{1}^{m-1} \cos m\left(\theta-\theta_{2}\right)}{m+b} .
$$

Hieraus folgt, wenn $\operatorname{man} \frac{\mathrm{I}}{r_{\mathrm{I}}}=\mathrm{I}+s_{\mathrm{s}}$ setzt,

$$
\begin{aligned}
\frac{d R}{d r_{1}}+b R & =\int_{\theta=0}^{\theta=2 \pi} F_{0} \cdot\left\{\frac{\mathrm{I}}{2 \pi}+\frac{\mathrm{I}}{\pi} \sum_{m}^{\mathrm{I}, \infty} r_{\mathrm{z}}^{m} \cos m\left(\theta-\theta_{\mathrm{I}}\right)\left(\mathrm{I}+s_{\mathrm{I}}\left(\mathrm{I}-\frac{b}{m+b}\right)\right)\right\} d \theta \\
& =\left(\mathrm{I}+s_{\mathrm{1}}\right) \int_{0}^{2 \pi} F_{\mathrm{O}} \cdot\left\{\frac{\mathrm{I}}{2 \pi}+\frac{\mathrm{I}}{\pi} \sum_{m}^{\mathrm{r}, \infty} r_{\mathrm{I}}^{m} \cos m\left(\theta-\theta_{\mathrm{x}}\right)\right\} d \theta \\
& -b s_{1} \int_{0}^{2 \pi} F_{\mathrm{O}} \cdot\left\{\frac{\mathrm{I}}{2 \pi}+\frac{\mathrm{I}}{\pi} \sum_{m}^{1, x} \frac{r_{1}^{m} \cos m\left(\theta-\theta_{\mathrm{I}}\right)}{m+b}\right\} d \theta .
\end{aligned}
$$

Jetzt lassen wir $r_{1}$ gegen I konvergieren oder $s_{1}$ unendlich abnehmen. Dann sind die Glieder der Reihe

$$
T=s_{i} \sum_{m}^{\mathrm{i}, \infty} \frac{r_{1}^{m} \cos m\left(\theta-\theta_{1}\right)}{m+b}
$$

absolut kleiner als die entsprechenden der Reihe

$$
s_{1} \sum_{m}^{\mathrm{r}, \infty} \frac{r_{\mathrm{I}}^{m}}{m}=-s_{\mathrm{I}} \log \left(\mathrm{I}-s_{\mathrm{s}}\right)
$$


und die Summe dieser wird mit $s_{\mathrm{I}}$ unendlich klein; dasselbe gilt also von der Summe $T$ unabhängig von dem Wert des Winkels $\theta$, mithin auch von dem zweiten Integral in dem Ausdruck $\frac{d R}{d r}+b R$. Da nun ferner aus der Theorie des Poisson'schen Integrals wohlbekannt ist, dass, wenn die Funktion $F$ o stetig ist, die Gleichung

gilt, so ist gezeigt

$$
\lim _{r_{\mathrm{I}}=\mathrm{r}} \int_{0}^{2 \pi} F_{\mathrm{o}} \cdot\left\{\frac{\mathrm{I}}{2 \pi}+\frac{\mathrm{I}}{\pi} \sum_{m}^{\mathrm{I}, \infty} r_{\mathrm{I}}^{m} \cos m\left(\theta-\theta_{\mathrm{I}}\right)\right\} d \theta=F_{\mathrm{I}}
$$

Die Grösse

$$
\lim _{r_{\mathrm{r}}=\mathrm{I}}\left(\frac{d R}{d r_{1}}+b R\right)=F_{\mathrm{I}}
$$

$$
\int F \text { o. } K(\mathrm{o}, \mathrm{I}) d l_{\mathrm{o}}
$$

ist nun aber offenbar, auch wenn man längs des Kreises $r=\mathrm{I}$ integriert, von der Natur eines logarithmischen Potentials, dessen in der Richtung eines Radius genommene Ableitung, wenn man von einer bestimmten Seite her auf die belegte Kreislinie herangeht, stetig in einen endlichen Grenzwert übergeht. Somit gilt die Gleichung

$$
\lim _{r_{1}=1}\left(\frac{d R}{d r_{1}}+b R\right)=-\frac{d R}{d n}+\left.b R\right|^{r_{1}=1}
$$

und es ist klar, dass die rechts stehende Grösse den willkürlich vorgeschriebenen Wert

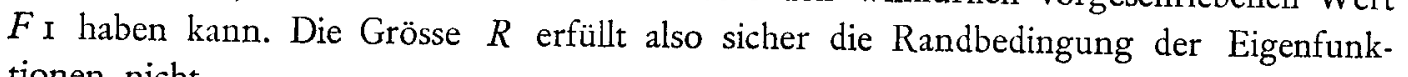
tionen nicht.

Um diese Tatsache für uns interessant zu machen, muss noch gezeigt werden, dass man $R$ auf die FourIER'sche Art nach den Eigenfunktionem $\varphi_{m \mu}, \bar{\varphi}_{m \mu}$ entwickeln kann. $\mathrm{Zu}$ diesem $\mathrm{Z}_{\text {weck }}$ gehen wir davon aus, dass die Reihe

$$
K(\mathrm{o}, \mathrm{I})=\frac{\mathrm{I}}{2 \pi b}+\sum_{m}^{\mathrm{r}, \infty} \frac{r_{1}^{m} \cos m\left(\theta-\theta_{1}\right)}{\pi(m+b)}
$$

offenbar gliedweise nach $\theta$ integriert werden kann, auch wenn man $r_{1}=1$ setzt; sie behält diese Eigenschaft, wenn man sie mit einer Funktion von $\theta$ wie etwa $F$ o multipliziert. Man kann also zunächst ähnlich wie in $₫ 3$ setzen

$$
\int F \mathrm{o} . K(\mathrm{o}, \mathrm{I}) d l_{\mathrm{o}}=\frac{\mathrm{I}}{\pi} \sum_{m}^{0, \infty} \int_{\left(r_{0}=1\right)} K\left(r, r_{\mathrm{l}}\right)_{m} F_{\mathrm{o}} \cdot \cos m\left(\theta-\theta_{\mathrm{s}}\right) d l_{\mathrm{o}},
$$

und findet dann, indem man die in $\$ 3$ gegebene Entwicklung wiederholt, dass in der Tat unser Ausdruck auf der ganzen Kreisfäche $G$ in der angegebenen Weise nach den Eigenfunktionen $\varphi, \bar{\varphi}$ entwickelt werden kann.

Ein wenig anders liegen die Verhältnisse bei der Annahme $b=\infty$. Hier verschwinden alle Eigenfunktionen auf dem Rande $r=\mathrm{I}$; eine nach ihnen fortschreitende Reihe wird also im strengen Sinne des Wortes immer die Randbedingung der Eigenfunktionen erfüllen. Aber es lässt sich hier eine ähnliche Erscheinung nachweisen, wie sie bei den FourIER'schen Reihen im engeren Sinne des Wortes die Reihe

$$
\sum_{m}^{\mathrm{s}, \infty} \frac{\sin m x}{m}
$$


darbietet. Dazu führt der Ausdruck

$$
S=S \mathrm{I}=-\int_{\left(r_{\mathrm{o}}=1\right)} F_{\mathrm{o}} \cdot \frac{d K(\mathrm{o}, \mathrm{I})}{d r_{\mathrm{o}}} d l_{\mathrm{o}} \text {. }
$$

Gehört nämlich der Punkt I dem Kreise $r=\mathrm{I}$ nicht an, so folgt aus $₫ 4$ unmittelbar, dass man $S_{\mathrm{I}}$ in eine nach FourIER'scher Weise gebildete Reihe entwickeln kann. Diese bietet nun, wenn man den Punkt I gegen den Kreis $r=\mathrm{I}$ heranrücken lässt, eine Unstetigkeit dar. Man hat nämlich offenbar die Gleichung

hieraus folgt

$$
\begin{gathered}
\frac{d K(\mathrm{O}, \mathrm{I})}{d r}=\frac{d}{d r}\left[\frac{\mathrm{I}}{2 \pi b}-\frac{\log r}{2 \pi}+\sum_{m}^{\mathrm{I}, \infty} \frac{\cos m\left(\theta-\theta_{\mathrm{I}}\right)}{2 \pi m}\left(\left(\frac{r_{\mathrm{I}}}{r}\right)^{m}-r^{m} r_{\mathrm{I}}^{m}\right)\right] \\
=-\frac{\mathrm{I}}{2 \pi}-\sum_{m}^{\mathrm{I}, \infty \infty} \frac{r_{\mathrm{I}}^{m} \cos m\left(\theta-\theta_{\mathrm{I}}\right)}{\pi} ;
\end{gathered}
$$

$$
S_{\mathrm{I}}=\int_{0}^{2 \pi} F_{\mathrm{O}} \cdot\left\{\frac{\mathrm{I}}{2 \pi}+\frac{\mathrm{I}}{\pi} \sum_{m}^{\mathrm{r}, \infty} r_{\mathrm{I}}^{m} \cos m\left(\theta-\theta_{\mathrm{I}}\right)\right\} d \theta .
$$

Der Grenzwert dieser Grösse ist aber, wenn man $r_{1}$ gegen I konvergicren und die Stelle $\mathrm{I}$ in die Lage 2 rücken lässt, wiederum nach der Theorie des PoIsson'schen Inf̧egrals

$$
\lim _{r_{1}=1} S=F_{2},
$$

also eine im allgemeinen von Null verschiedene willkürliche Funltion des Ortes auf dem Kreise $r=1$. Damit ist die Unstetigkeit der Reihe $S$ erwiesen. Setzt man noch

$$
A_{m \mu}=-\int_{0}^{2 \pi} F \mathrm{o} \cdot \frac{d \varphi_{m \mu} \mathrm{o}}{d r} d \theta, \quad \bar{A}_{m \mu}=-\int_{0}^{2 \pi} F \mathrm{o} \cdot \frac{d \bar{\varphi}_{m \mu} \mathrm{o}}{d r} d \theta
$$

so gilt im Innern des Gebiets $G$ die Gleichung

$$
S=\sum_{m}^{\circ, \infty} \sum_{\mu}^{1, \infty}\left(A_{m \mu} \varphi_{m \mu}+\bar{A}_{m \mu} \bar{\varphi}_{m \mu}\right)
$$

und man verifiziert leicht die Beziehungen

$$
A_{m \mu}=\int_{G} S_{p_{m \mu}} d s, \quad \bar{A}_{m \mu}=\int_{G} S_{\Phi_{m \mu}} d s
$$

$\$ 6$.

\section{Allgemeine Ergebnisse.}

Aus den speziellen, auf die Grössen $P, Q, R$ bezüglichen Resultaten folgen unmittelbar die allgemeineren, die das Ziel der Untersuchung bilden.

Auf der Kreisfläche $G$ seien eine beliebige Anzahl sich selbst und einander nicht schneidender Kurven $L$ gegeben, die entweder geschlossen sind oder von einem Punkt des Randes zu einem anderen gehen; jede von ihnen möge entweder durch $L_{p}$ oder durch $L_{q}$ oder durch beide Charaktere zugleich bezeichnet werden. $\Phi$ sei in Gebiet $G$ eine Funktion des Ortes, die an den Linien $L_{p}$ unstetige erste Ableitungen besitzt, an 
den Linien $L_{q}$ selbst unstetig ist, und am Rande des Gebiets $G$ die Randbedingung der Eigenfunktionen nicht erfüllt, im übrigen aber mit ihren Ableitungen erster Ordnung auf dem Gebiet $G$ stetig ist, während die Grösse $\mathbf{\perp} \Phi$ und ihr Quadrat nur integrierbar $\mathrm{zu}$ sein brauchen. Im Falle $b=0$ sei

$$
\int_{G} \Phi d s=0 .
$$

Die Unstetigkeiten an den Linien $L_{p}$ seien durch Gleichungen von der Form

$$
\frac{\partial \Phi}{\partial n}+\frac{\partial \Phi}{\partial n^{\prime}}=-F_{p}
$$

an den Linien $L_{q}$ durch Gleichungen

$$
\boldsymbol{\Phi}^{\prime}-\boldsymbol{\Phi}^{\mathrm{o}}=F_{q}
$$

definiert, in denen durch $n$ und $n^{\prime}$ die beiden Richtungen der Normale der Kurve $L_{p}$, durch $\Phi^{\prime}$ und $\Phi^{\circ}$ die Grenzwerte bezeichnet sind, denen die Funktion $\Phi$ zustrebt, wenn man sich einem Punkte einer Kurve $L_{q}$ von der einen oder anderen Seite her annähert. $F_{p}$ und $F_{q}$ seien Funktionen des Ortes auf jeder der Linien $L_{p}$ und $L_{q}$, die am Rande des Gebiets $G$ verschwinden; fallen $L_{p}$ und $L_{q}$ zusammen, so brauchen die zugehörigen Grössen $F_{p}$ und $F_{o}$ nicht übereinzustimmen. Für die Linien $L$ und die Grössen $F$ werden ferner Stetigkeitseigenschaften gefordert, die in $\ 3$ genauer formuliert sind, im wesentlichen aber bedeuten, dass $L$ und $F$ einigermassen vernünftige Kurven und Funktionen im Sinne JACOBI's sind, d. h. dass man einigemale differenzieren darf und keine der betrachteten Variabeln einen bestimmten Wert unendlich oft annimmt.

Unter diesen Voraussetzungen kann für jede Linie $L_{b}$ nach $\$ 3$ eine Grösse $P$, für jede Linie $L_{0}$ nach $\ 4$ eine Grösse $Q$ konstruiert werden, indem man für die in den zitierten Paragraphen benutzte Grösse $F$ die zu der betreffenden Linie $L_{n}$ oder $L_{n}$ gehörige Grösse $F_{p}$ oder $F_{q}$ nimmt. $P$ und $Q$ sind dann Funktionen des Ortes auf der Fläche $G$; an den Kurven $L_{p}$ gelten die Gleichungen

$$
\frac{\partial P}{\partial n}+\frac{\partial P}{\partial n^{\prime}}=-F_{p}
$$

an den Kurven $L$, die Gleichungen

$$
Q^{\prime}-Q^{\circ}=F_{q},
$$

wobei die Symbole $Q^{\prime}, Q^{\circ}$ ähnlich wie $\Phi^{\prime}, \Phi^{\circ}$ zu verstehen sind. Hịeraus folgt, dass die Grösse

$$
\Phi-\Sigma P-\sum Q
$$

in der über alle Linien $L_{p}$ und $L_{a}$ summiert werde, auf der ganzen Fläche $G$ stetig ist und an jeder Kurve $L$ stetige normale Ableitungen besitzt.

Ferner kann eine Funktion $R$ nach $\ 5$ gebildet werden, indem man bei endlichen Werten von $b$

$$
F=-\frac{d \Phi}{d r}-\left.b \Phi\right|^{r=\mathbf{r}}
$$


im Falle $b=\infty$ aber

$$
F=\left.\Phi\right|^{r=1}
$$

setzt. Dann erfüllt die Differenz $\Phi-R$ die Randbedingungen der Eigenfunktionen, ebenso, da dies auch von $P$ und $Q$ gilt, der Ausdruck

$$
\Theta=\Phi-R-\sum P-\sum Q
$$

der dieselben Stetigkeitseigenschaften wie $\Theta+R$ hat. Dabei ist die Grösse

$$
f \circ=\Delta \theta
$$

und ihr Quadrat ebenso integrierbar wie $\Delta \Phi$ und $(\Delta \Phi)^{2}$, da die Grössen $P, Q, R$ nur die Singularitäten von Linienpotentialen darbieten. Auf Grund dieser Eigenschaften kann die Grösse $\Theta$ auf die Fourier'sche Art nach den zur Kreisfläche $G$ gehörenden Eigenfunktionen $\varphi, \bar{\varphi}$ entwickelt werden; wir beweisen dies, indem wir den zitierten Satz von STEkLoFF ein wenig verallgemeinern.

Zu diesem Zweck werde jede Kurve $L$ in einen beliebig schmalen Streifen eingeschlossen, den die Kurven $L^{\prime}$ und $L^{\prime \prime}$ begrenzen, die man sich etwa als Parallelkurven der Linie $L$ vorstellen kann; vor den Kreis $r=I$ werde ein Kreisring von der beliebig kleinen Breite $\varepsilon$ gelegt und die Stelle $\mathrm{I}$, die zunächst im Innern von $G$ und nicht auf einer Kurve $L$ liege, werde mit einem beliebig kleinen Kreise $k$ umgeben, in dessen Mittelpunkt sie liegt. Schliesst man alle diese Gebiete von $G$ aus, so bleibt ein Gebiet $G_{1}$ übrig, auf das man die Gauss'sche Integraltransformation

$$
\int(u \Delta v-v \Delta u) d s=\int d l\left(v \frac{d u}{d n}-u \frac{d v}{d n}\right)
$$

anwenden kann, indem man

$$
u=K(\mathrm{o}, \mathrm{I}), \quad v=\theta_{\mathrm{o}}
$$

setzt und rechts über die Kurvenpaare $L^{\prime}, L^{\prime \prime}$, den Kreis $r=\mathbf{I}-\varepsilon$ und den Kreis $k$ integriert, wobei die Normale $n$ nach dem Innern des Gebiets $G_{1}$ hineinzeige. Man sieht dann unmittelbar, da an den Linien $L$ beide Grössen $u, v$ und ihre normalen Ableitungen stetig sind, dass die Summanden

$$
\int_{L^{\prime}}\left(v \frac{d u}{d n}-u \frac{d v}{d n}\right) d l+\int_{L^{\prime \prime}}\left(v \frac{d u}{d n}-u \frac{d v}{d n}\right) d l
$$

erstreckt über ein Paar zusammengehöriger Kurven $L^{\prime}, L^{\prime \prime}$, eine der Null um so näher liegende Summe ergeben, je schmaler der von $L^{\prime}$ und $L^{\prime \prime}$ eingeschlossene Streifen ist, dass auch das auf den Kreis $r=\mathrm{I}-\varepsilon$ bezügliche Integral beliebig klein gemacht werden kann, da die Grössen $u$ und $v$ beide die Randbedingung der Eigenfunktionen erfüllen, also die Grösse

$$
v \frac{d u}{d n}-\left.u \frac{d v}{d n}\right|^{r=1-\varepsilon}
$$

mit $\varepsilon$ unendlich klein wird. Da ferner das über den Kreis $k$ erstreckte Integral annähernd durch die Formel

$$
\int_{k} \Theta \mathrm{o} \cdot \frac{d}{d n}\left(\frac{\mathrm{I}}{2 \pi} \log \frac{\mathrm{I}}{\mathrm{P}_{\mathrm{OI}}}\right) \cdot d l_{\mathrm{o}}=-\Theta \mathrm{I}
$$

Rend. Circ. Matem. Palermo, t. XXVII ( ${ }^{\circ}$ sem, 1909 ). - Stampato il 25 novembre 1908. 
dargestellt wird, konvergiert es offenbar gegen $-\Theta \mathrm{I}$, wenn der Radius dieses Kreises unbegrenzt abnimmt; endlich verschwindet $\Delta u$ bei der Annahme $b>0$ nach $₫ 2$ und man erhält, indem man $G_{\imath}$ in $G$ übergehen lässt, die Formel

$$
\int_{G} f o \cdot K(\mathrm{o}, \mathrm{I}) d s_{\mathrm{o}}=-\Theta_{\mathrm{I}} \text {. }
$$

Dasselbe Resultat ergibt sich im Falle $b=0$ leicht aus den Gleichungen

$$
\Delta u=\frac{\mathrm{I}}{\pi}, \quad \int_{G} \Phi d s=0 .
$$

Die bisher geltende Voraussetzung, die Stelle I liege auf keiner der Kurven $L$ und $r=\mathrm{I}$, kann jetzt aufgehoben werden, da beide Seiten der erhaltenen Formel als Funktionen der Stelle I auf dem ganzen Gebiet $G$ mit Einschluss des Randes stetig sind.

Die Funktion $\Theta$ ist hiermit auf diejenige Form gebracht, aus der die Theorie der Integralgleichungen ${ }^{10}$ ) $\mathrm{zu}$ schliessen erlaubt, dass man $\Theta$ nach den Eigenfunktionen $\varphi_{m \mu}, \bar{\varphi}_{m \mu \mu}$ auf die Fourier'sche Art entwickeln kann.

Auf eben diese Art lassen sich nun nach den $\$ \$ 3,4,5$ die Grössen $P, Q, R$ entwickeln; die erhaltenen Reihen gelten auch auf den Unstetigkeitslinien $L$ mit einziger Ausnahme derjenigen Punkte der Linien $L_{q}$, in denen die radiale Polarkoordinate $r$ ein Extremum erreicht. Von diesen abgesehen stellt nach $\$ 4$ die für irgend eine Grösse $Q$ erhaltene Reihe auf der Kurve $L_{q}$ den Wert

$$
\frac{1}{2}\left(Q^{\circ}+Q^{\prime}\right)
$$

dar, der dem Wert von $Q$ an der betrachteten Stelle der Kurve $L_{q}$ gleich ist, da ja die Grösse $Q$ unstetig ist wie das logarithmische Potential einer Doppellinie. Aus diesen Tatsachen und der Identität

$$
\Phi=\Theta+R+\sum P+\Sigma Q
$$

folgt sofort, dass auch die Grösse $\Phi$ auf die Fourier'sche Art nach den Eigenfunktionen $\varphi, \bar{\varphi}$ entwickelt werden kann, d.h. dass in der Bezeichnung

die Gleichung

$$
A_{m \mu}=\int_{G} \Phi \varphi_{m \mu} d s, \quad \bar{A}_{m \mu}=\int_{G} \Phi \bar{\varphi}_{m \mu} d s
$$

$$
\Phi=\sum_{m}^{0, \infty} \sum_{\mu}^{\mathrm{x}, \infty}\left(A_{m \mu} \varphi_{m \mu}+\bar{A}_{m \mu} \bar{\varphi}_{m \mu}\right)
$$

gilt, und zwar so, dass der Wert dieser Reihe auf den Unstetigkeitslinien $L_{q}$ im allgemeinen

$$
\frac{1}{2}\left(\Phi^{\circ}+\Phi^{\prime}\right)
$$

10) Stekloff, Théorie générale des fonctions fondamentales [Annales de la Faculté des Sciences de l'Université de Toulouse, Ser. II, Bd. VI (1904), S. 35[-475], S. 423. - HrLbert, Grundzüge einer allgemeinen Theorie der linearen Integralgleichungen (Erste Mitteilung) [Nachrichten von der Königl. Gesellschaft der Wissenschaften zu Göttingen, Mathematisch-physikalische Klasse, Jahrgang 1904, S. 49-91], S. 75. - Schmidt, S. 452, 459 der in Anm. 5) zitierten Abhandlung. 
ist. Nur in den Punkten, in denen $r$ auf einer Linie $L_{q}$ ein Extremum erreicht oder auch eine endliche Strecke hindurch konstant bleibt, ist es anders. Hier stellt die für $Q$ erhaltene Reihe einen der Werte $Q^{\circ}$ und $Q^{\prime}$ dar, mithin die für $\Phi$ gebildete einen der Werte $\boldsymbol{\Phi}^{\circ}$ und $\boldsymbol{\Phi}^{\prime}$.

Das erhaltene Resultat ist offenbar einem bekannten Satze über das Verhalten der gewöhnlichen FourieR'schen Reihe in den Unstetigkeitsstellen der dargestellten Funktion verwandt; es genügt an das Dirichlet'sche Symbol

zu erinnern.

$$
\frac{x}{2}\{f(x+0)+f(x-0)\}
$$

Sieht man übrigens davon ab, den Wert der nach den Eigenfunktionen $\varphi, \bar{\varphi}$ fortschreitenden Reihe in den Unstetigkeitslinien zu bestimmen, so fallen grosse Schwierigkeiten weg und der grösste Teil unserer Resultate lässt sich auf beliebig gestaltete Gebiete $G$ übertragen. Die bezeichnete besondere Frage scheint aber Untersuchungen zu erfordern, die mit der besonderen Natur der betrachteten Funktionen $\varphi, \bar{\varphi}$ zusammenhängen; im allgemeinen dürfte sie nur gefördert werden können, wenn es gelänge, die Eigenfunktionen eines beliebigen Gebietes durch die eines genauer untersuchten, z. B. der Kreisfläche, asymptotisch darzustellen.

Breslau, den 27. Mai 1908.

A DOLF KNESER. 